\title{
On Maximum Likelihood Estimation of Clock Offset and Skew in Networks With Exponential Delays
}

\author{
Qasim M. Chaudhari, Erchin Serpedin, and Khalid Qaraqe, Student Member, IEEE
}

\begin{abstract}
Clock synchronization represents a crucial element in the operation of wireless sensor networks (WSNs). For any general time synchronization protocol involving a two-way message exchange mechanism, e.g., timing synch protocol for sensor networks (TPSN) [see S. Ganeriwal, R. Kumar, and M. B. Srivastava, "Timing Synch Protocol for Sensor Networks," in Proceedings of the First International Conference on Embedded Network Sensor Systems," 2003, pp. 138-149], the maximum likelihood estimate (MLE) for clock offset under the exponential delay model was derived in [D. R. Jeske, "On the Maximum Likelihood Estimation of Clock Offset," IEEE TrANSACTIONS ON COMMUNICATIONS, vol. 53, no. 1, pp. 53-54, January 2005] assuming no clock skew between the nodes. Since all practical clocks are running at different rates with respect to each other, the skew correction becomes important for achieving long term synchronization since it results in the reduction of the number of message exchanges and hence minimization of power consumption. In this paper, the joint MLE of clock offset and skew under the exponential delay model for a two way timing message exchange mechanism and the corresponding algorithms for finding these estimates are presented. Since any time synchronization protocol involves real time message exchanges between the sensor nodes, ML estimates for other synchronization protocols can be derived by employing a similar procedure. In addition, due to the computational complexity of the MLE, a simple, computationally efficient and easy to implement algorithm is presented as an alternative to the ML estimator which particularly suits the low power demanding regime of wireless sensor networks.
\end{abstract}

Index Terms-Clock synchronization, maximum likelihood estimation (MLE), wireless sensor network (WSN).

\section{INTRODUCTION}

$\mathbf{R}$ ECENT technological advances have made it possible to design miniature devices (sensors) capable of performing onboard sensing, computing and communication tasks. A WSN consists of a large number of such tiny devices, called nodes, that are connected in an ad hoc manner without assuming any centralized infrastructure [3]. Today WSNs are increasingly gaining importance due to their applicability in various fields such as military surveillance, environmental monitoring, traffic monitoring and control, acoustic and seismic detection, industrial processes monitoring, etc. Since the WSN nodes are deployed in an ad hoc fashion and mostly left without

Manuscript received March 4, 2007; revised September 18, 2007. The associate editor coordinating the review of this manuscript and approving it for publication was Dr. Gerald Matz.

Q. M. Chaudhari and E. Serpedin are with the Department of Electrical and Computer Engineering, Texas A\&M University, College Station, TX 778433128 USA (e-mail: qasim@ece.tamu.edu; serpedin@ece.tamu.edu).

K. Qaraqe is with the Department of Electrical Engineering, Texas A\&M University at Qatar, Doha 23874, Qatar (e-mail: khalid.qaraqe@qatar.tamu.edu).

Digital Object Identifier 10.1109/TSP.2007.910536 any maintenance and battery replacement for their lifetimes, they are usually cheap and hence unreliable. Therefore, all the design aspects of a sensor network concentrate on minimizing energy utilization [4]-[6].

The operation of a WSN requires all its nodes be synchronized to a common time scale. Clock synchronization is important since it enables all the nodes to assume efficient duty cycling operation, i.e., coordinated sleep and wake up modes, which hugely boosts the lifetimes of the nodes due to the minimal power consumption during the sleep mode. Time synchronization is also important for object/phenomenon localization and tracking because different sensors recording the position and time of the object/phenomenon must be synchronized for reliable estimation. The benefits associated with deploying time division multiple access (TDMA) scheme can also be gained in a synchronized network. Clock synchronization is also important for data fusion so that the voice, video or environmental data from different sensors can be integrated and processed in a meaningful way [6]. Finally, many security protocols require the WSN nodes to timestamp their messages.

There are a few methods through which the accuracy of the nodes' clocks can be improved, e.g., using GPS to synchronize the hardware clocks to a global reference, using precise clock boards for the nodes, etc. But these solutions prove to be fairly expensive or inappropriate when the nodes have to be low-cost and energy efficient. In addition, the sensor nodes may be left unattended for a long period of time, e.g., on the ocean floor or in deep space. Also, the conventional network synchronization protocols can not be employed due to the WSN constraints mentioned above [7]. Hence, there is a need for time synchronization protocols specifically designed to the characteristics of WSNs to make them operate under a common time scale.

Time synchronization in WSNs requires designing a protocol in which the nodes exchange messages with each other to adjust their clocks to a common reference. At the same time, it is highly desirable to extract information about their relative frequency, called clock skew, from the same set of message exchange, because imperfections in quartz crystals and environmental conditions cause different nodes to run at different frequencies. Clock skew adjustment guarantees not only a more accurately synchronized network, but also helps in maintaining this synchronization for a longer period. Hence, it significantly reduces the resynchronization period, i.e., the time interval after which the clock difference among the nodes exceeds some set limits and the network has to resynchronize itself, resulting in tremendous reduction in communication overheads and corresponding energy savings for the whole network. 


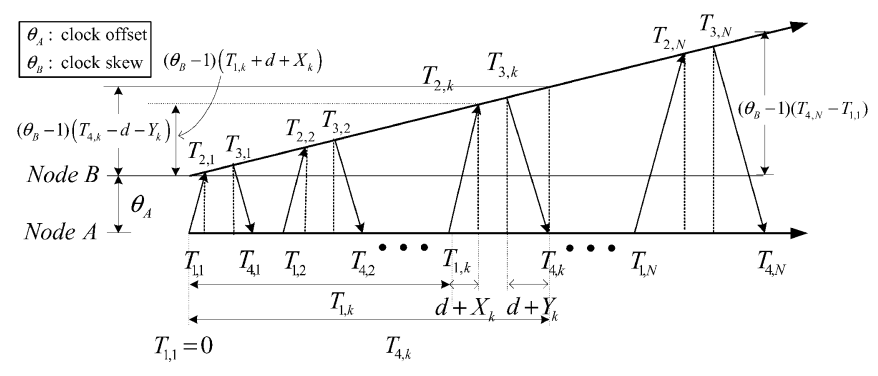

Fig. 1. Model for two way timing message exchange involving $N$ such pairs.

\section{RELATED WORK}

In the Internet, the network time protocol (NTP) [10] is used to provide distributed synchronization including adjusting the frequency of each node's oscillator. NTP synchronizes computer clocks in a hierarchical way by using primary and secondary time servers but it is not suited for WSNs because it does not take into account the energy consumption and bandwidth constraints. In addition, it is designed for continuous operation in the background at low rates.

To deal efficiently with the specific requirements associated with the long-term operation of WSNs, quite a few synchronization protocols have been designed in the past few years. Reference broadcast synchronization (RBS) [11] is a pioneering work based on the post-facto receiver-receiver synchronization. In RBS, a reference broadcast message is sent by a node to two or more neighboring nodes which record their own local clocks at the reception of broadcasted message. After collecting a few readings, the nodes exchange their observations and a linear regression approach is used to estimate their relative clock offset and skew. Timing synch protocol for sensor networks (TPSN) [1] is a conventional sender-receiver protocol which assumes two operational stages: the level discovery phase followed by the synchronization phase. During the level discovery phase, WSN is organized in the form of a spanning tree, and the global synchronization is achieved by enabling each node to get synchronized with its parent (the node located in the adjacent upper level) by means of a message exchange mechanism depicted in Fig. 1 through adjusting only its clock offset. Timing synchronization protocol for high latency acoustic networks (TSHL) [13] combines both of these approaches in two stages. The first stage is similar to RBS while the second stage is similar to TPSN, and it is particularly suitable for networks involving high message delays, e.g., underwater acoustic networks. Flooding time synchronization protocol (FTSP) [12] also combines the two approaches in the sense that the beacon node sends its timestamps within the reference broadcast messages.

All of the above mentioned protocols have their own benefits and limitations. Choosing a protocol which corrects only the clock offset (such as TPSN [1]) results in more utilization of power since synchronization has to be done frequently at regular intervals to prevent the clock skew drift the two clocks too far apart. For example, re-synchronization must be performed after every few minutes in TPSN for applications using Berkeley motes $^{1}$. On the other hand, an assumption of simultaneous re-

\footnotetext{
${ }^{1}$ http://webs.cs.berkeley.edu/tos/
}

ception of reference broadcasts is necessary in protocols which correct both the clock offset and skew (such as RBS [11] and FTSP [12]), which is not only a simplification of the correct model but also not applicable in some cases, e.g., in underwater acoustic sensor networks [13]. As argued in [14] and motivated in detail in [13], TPSN could represent a very efficient synchronization protocol if the clock skew information can also be extracted from the same message exchange mechanism without any additional communication overheads. This is exactly the target of this paper.

In 2002, [8] presented a detailed analysis of clock offset estimation assuming a symmetric exponential delay model. It was implicitly argued that for a known fixed delay $d$ and exponential delay parameter $\alpha$, the MLE of clock offset $\theta_{A}$ does not exist because the likelihood function does not possess a unique maximum with respect to $\theta_{A}$. However, in 2005, it was proved by [2] that for $d$ unknown, irrespective of $\alpha$ being known or unknown, the MLE of $\theta_{A}$ does exist and coincides with a previously proposed estimator in [9]. In 2006, the Cramér-Rao lower bound (CRLB) for this estimator was derived by [14]. However, the problem of jointly estimating the clock offset $\theta_{A}$ and clock skew $\theta_{B}$ (with $d$ known or unknown) in the exponential delay case remained open and posed quite a serious challenge.

In this paper, the MLEs of both clock offset and skew are derived for the exponential delay model and the corresponding algorithms for finding those estimates are presented in detail. It should be noticed that this methodology can be employed to any time synchronization protocol involving a two way message exchange mechanism between two nodes. Estimation of clock skew is very important for the reasons described in the previous section, and finding the MLE is desirable due to its optimal properties in the presence of a large number of observations (i.e., unbiasedness, asymptotic efficiency and consistency) [20]. In addition, a novel algorithm is proposed to estimate the required parameters which significantly reduces the computational load on the nodes. The price paid is some degradation in the performance of the estimator.

The rest of this paper is organized as follows. The model for the TPSN protocol assuming exponential delays is explained in Section III. Section IV presents the Maximum Likelihood Estimates of the clock offset, skew and fixed delay (where unknown). Section $\mathrm{V}$ proposes a novel simplified algorithm requiring much less computational complexity than the MLE. Computer simulations for assessing the relative performance of the proposed estimator are presented in Section VI. Section VII presents a comparison of the computational complexity of each algorithm. Finally, Section VII concludes the paper.

\section{PAIRWISE SYNCHRONIZATION PROTOCOL}

The timing message exchange mechanism between two nodes presenting both clock offset and skew is depicted in Fig. 1. Node A sends the synchronization message to Node B with the its current timestamp $T_{1, k}$ (although it is not required, timestamping in the MAC layer increases the accuracy, as suggested by [1]). Node $\mathrm{B}$ records its current time $T_{2, k}$ at the reception of this message, then completes the second round of this message exchange mechanism by sending at time $T_{3, k}$ a synchronization message to Node A containing $T_{2, k}$ and $T_{3, k}$. Node 
A timestamps the reception time of the message sent by Node $\mathrm{B}$ as $T_{4, k}$ (see Fig. 1). Hence, at the end of synchronization cycle of exchanging $N$ such rounds, Node A has a set of timestamps $\left\{T_{1, k}, T_{2, k}, T_{3, k}, T_{4, k}\right\}, k=1, \cdots, N$. Note that $T_{1,1}$ is considered to be the reference time, and hence every reading $\left\{T_{1, k}, T_{2, k}, T_{3, k}, T_{4, k}\right\}$ is actually the difference between the recorded time and $T_{1,1}$. Therefore, this model can be represented as

$$
\begin{aligned}
& T_{2, k}=\left(T_{1, k}+d+X_{k}\right) \theta_{B}+\theta_{A} \\
& T_{3, k}=\left(T_{4, k}-d-Y_{k}\right) \theta_{B}+\theta_{A}
\end{aligned}
$$

where $\theta_{A}$ and $\theta_{B}$ are the clock offset and skew, respectively, of Node B with respect to Node A, $d$ stands for the fixed portion of delay in the transmission of message from one node to another, e.g., the sum of transmission time, propagation delay, reception time, etc., and $X_{k}$ and $Y_{k}$ are variable portions of delay assumed to be independent and identically distributed random variables from an exponential distribution with the same mean $\alpha$. Modeling of network delays in WSN seems to be a challenging task [17]. Several probability distribution function (pdf) models for random queuing delays have been proposed so far, the most widely used of which are exponential, Gamma, and log-normal distributions [15], [16], [18]. Amongst them, the exponential distribution fits quite well several applications [19]. Also, a single-server $\mathrm{M} / \mathrm{M} / 1$ queue can fittingly represent the cumulative link delay for point-to-point Hypothetical Reference Connection, where the random delays are independently modeled as exponential random variables [8]. In addition, [9] experimentally demonstrated the superiority of the Minimum Link Delay (MnLD) algorithm among the various algorithms proposed by [8], which was mathematically proved by [2] assuming exponential delays, thus confirming that the exponential delay assumption matches really well with the experimental observations.

\section{MAXIMUM LiKeLIHOOD Estimation}

From (1) and (2), the general form of the likelihood function is given by

$$
\begin{array}{r}
L\left(\alpha, d, \theta_{B}, \theta_{A}\right) \\
=\alpha^{-2 N} \cdot \exp \left[-\frac{1}{\alpha}\left\{\sum_{k=1}^{N} \frac{T_{2, k}-T_{3, k}}{\theta_{B}}\right.\right. \\
\left.\left.-\sum_{k=1}^{N}\left(T_{1, k}-T_{4, k}\right)-2 N d\right\}\right] \\
\times \prod_{k=1}^{N} I\left[\frac{T_{2, k}-\theta_{A}}{\theta_{B}}-T_{1, k}-d \geq 0 ; T_{4, k}\right. \\
\left.-\frac{T_{3, k}-\theta_{A}}{\theta_{B}}-d \geq 0\right]
\end{array}
$$

where the indicator function $I[\cdot]$ is defined as

$$
I[\tau \geq 0]=\left\{\begin{array}{l}
1, \tau \geq 0 \\
0, \tau<0 .
\end{array}\right.
$$

Note that the $d$ is always positive since it represents the delay (fixed), while $\theta_{B}$ is also always positive because it has been realistically assumed that none of the clocks is either standing still $\left(\theta_{B}=0\right)$ or running backward $\left(\theta_{B}<0\right)$. An ideal value of $\theta_{B}=1$ means that the clock is running at the standard rate. Also, notice that when $\theta_{B}=1$, the MLE of clock offset $\theta_{A}$ was derived by [2] and takes the form

$$
\hat{\theta}_{A}=\frac{1}{2}\left[\min _{1 \leq k \leq N}\left(T_{2, k}-T_{1, k}\right)-\min _{1 \leq k \leq N}\left(T_{4, k}-T_{3, k}\right)\right] .
$$

From here onwards, without losing any generalization, we will assume that $\alpha$ is known. This is because even if $\alpha$ is unknown, due to the form of the reduced likelihood function $L\left(d, \theta_{B}, \theta_{A}\right)$ as shown in [2], the $\operatorname{MLE}\left(\hat{d}, \hat{\theta}_{B}, \hat{\theta}_{A}\right)$ remains the same. When $\theta_{B} \neq 1$, in maximizing the likelihood for this model over the set $\Theta=\left\{\left(d, \theta_{A}, \theta_{B}\right): d>0,-\infty<\theta_{A}<\infty, \theta_{B}>0\right\}$, four different cases will be considered:

Case I: $d$ known, $\theta_{A}$ known;

Case II: $d$ unknown, $\theta_{A}$ known;

Case III: $d$ known, $\theta_{A}$ unknown;

Case IV: $d$ unknown, $\theta_{A}$ unknown.

An important remark needs to be mentioned here. A preliminary examination of Cases I and II (i.e., when $\theta_{A}$ is known) is necessary because it gives insight into the shape of the support region over which the likelihood function is nonzero. As it is the case with exponential models, the MLEs for the location parameters will be found by taking effectively into account the boundary conditions. For the first two cases, the support of the likelihood region is a 2-D region and it is relatively easier to find the parameters on the boundary maximizing the likelihood function. Finding the MLEs for Cases III and IV (i.e., when $\theta_{A}$ is unknown) requires the visualization of the likelihood function support region in 3-D and getting a somewhat primitive knowledge of the 2-D support region for the likelihood function in Cases I and II greatly helps in preparing our intuition and solving the more complex 3-D optimization problem. Therefore, we next proceed with a stepwise approach by considering these four cases separately one-by-one.

\section{A. Case I: d Known, $\theta_{A}$ Known}

Without losing any generalization, the likelihood function in this case can be obtained by making $\theta_{A}=0$ in (3). From the form of the likelihood function, we can see that it is nonzero only over a certain support region defined by the limits of the indicator function $I[\cdot]$. Since $d$ is fixed and known, the set of constraints in (3), namely $d>0, \theta_{B}>0$ and

$$
\begin{aligned}
& d \leq \frac{T_{2, k}}{\theta_{B}}-T_{1, k}, \quad k=1, \ldots, N \\
& d \leq T_{4, k}-\frac{T_{3, k}}{\theta_{B}}, \quad k=1, \ldots, N
\end{aligned}
$$

can be equivalently put in the form

$$
\begin{aligned}
d & >0, \theta_{B}>0, \\
\frac{T_{3, k}}{T_{4, k}-d} & \leq \theta_{B} \leq \frac{T_{2, k}}{T_{1, k}+d}, \quad k=1, \ldots, N .
\end{aligned}
$$

Fig. 2 shows various upper-bounds (5) and (6) of the likelihood support region in the plane $\left(d, \theta_{B}\right)$, and the solid line is 


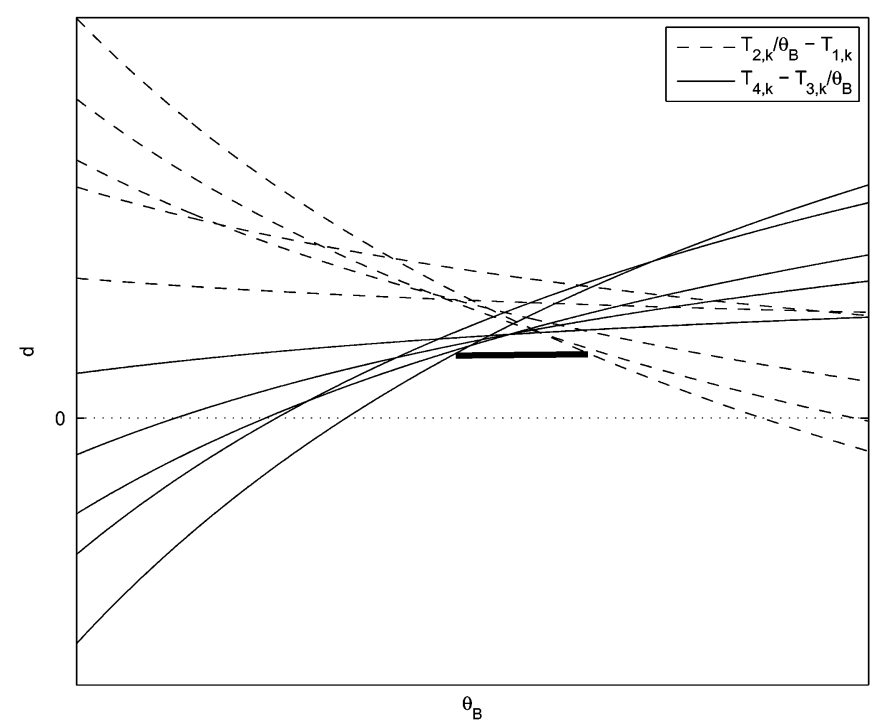

Fig. 2. Nonzero likelihood region shown as the solid line.

the region over which the likelihood function has to be maximized. It is evident from the figure that for a known fixed $d$, the likelihood function depends on the unknown $\theta_{B}$ only and is maximized by taking $\theta_{B}$ as small as possible. This is because the factor $\sum_{k=1}^{N}\left(T_{2, k}-T_{3, k}\right)$ in (3) is always negative. Therefore, the smallest value of $\theta_{B}$ over the solid line, as shown in Fig. 2, is the MLE $\hat{\theta}_{B}$, which coincides with one of the curves $d=T_{4, k}-T_{3, k} / \theta_{B}, k=1, \ldots, N$. Let $j(1 \leq j \leq N)$ denote the index of the curve on which the MLE is achieved. Thus, from (7), $j=\arg \max _{k}\left\{T_{3, k} /\left(T_{4, k}-d\right)\right\}$ and

$$
\hat{\theta}_{B}=\frac{T_{3, j}}{T_{4, j}-d} .
$$

The index $j$, which gives the set of timestamps $\left\{T_{3, j}, T_{4, j}\right\}$ required for finding the MLE, is the one which gives the minimum possible $\hat{\theta}_{B}$ over the allowable region. Since $d$ is known, we can find $j$, and hence the corresponding $\hat{\theta}_{B}$, by Algorithm 1 .

\section{Algorithm 1: Finding $\hat{\theta}_{B}$ for $d$ known, $\theta_{A}$ known \\ 1: Find $\theta_{B, k}=\left(T_{3, k}\right) /\left(T_{4, k}-d\right)$, for $k=1, \ldots, N$; \\ 2: $j=\arg \max _{k}\left\{\theta_{B, k}\right\}$; \\ 3: $\hat{\theta}_{B}=\hat{\theta}_{B, j}$;}

Algorithm 1 utilizes the fact that the solid line cuts all the curves $T_{4, k}-T_{3, k} / \theta_{B}, k=1, \ldots, N$ but the likelihood function is zero beyond its intersection with the first curve, which is the maximum of these intersections and therefore gives the MLE. Note that in doing so, a total number of $N$ values need to be compared. To simplify the exposition, in what follows we will use the terminology the curves $T_{4, k}-T_{3, k} / \theta_{B}, k=1, \ldots, N$, instead of the curves $d=T_{4, k}-T_{3, k} / \theta_{B}, k=1, \ldots, N$.

\section{B. Case II: d Unknown, $\theta_{A}$ Known}

The likelihood function in this case is similar to Case I, but with one major difference: the fixed delay $d$ is unknown. The shaded region in Fig. 3 is the subset of $\Theta$ over which the like-

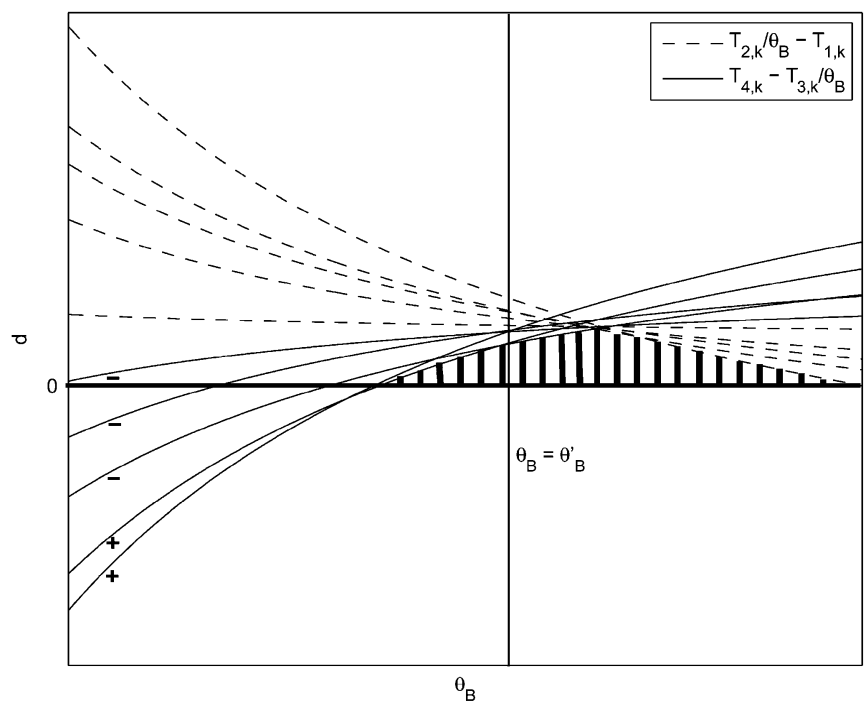

Fig. 3. Support region of the likelihood function shown as the shaded area. Also shown to the left in this figure is the sign of the term $2 N T_{3, j}-\sum_{k=1}^{N}\left(T_{3, k}-\right.$ $T_{2, k}$ ) for each $j=1, \ldots, N$.

lihood function is nonzero. It can be described in terms of the following constraints:

$$
\begin{aligned}
& d>0, \theta_{B}>0, \\
& d \leq \frac{T_{2, k}}{\theta_{B}}-T_{1, k}, \quad k=1, \ldots, N \\
& d \leq T_{4, k}-\frac{T_{3, k}}{\theta_{B}}, \quad k=1, \ldots, N .
\end{aligned}
$$

This likelihood function in (3) is maximized by making its argument

$$
\xi=\sum_{k=1}^{N} \frac{T_{2, k}-T_{3, k}}{\theta_{B}}-\sum_{k=1}^{N}\left(T_{1, k}-T_{4, k}\right)-2 N d
$$

as small as possible. Although Fig. 3 shows only the support region and not the likelihood function itself, $\xi$ can be linked to this figure by rewriting it in the form

$$
\xi=\sum_{k=1}^{N}\left(\frac{T_{2, k}}{\theta_{B}}-T_{1, k}\right)+\sum_{k=1}^{N}\left(T_{4, k}-\frac{T_{3, k}}{\theta_{B}}\right)-2 N d
$$

and noting that for any $\theta_{B}=\theta_{B}^{\prime}, \xi$ is the sum of the ordinates of all points on the curves $\left(T_{2, k} / \theta_{B}-T_{1, k}\right), k=1, \ldots, N$, and $\left(T_{4, k}-T_{3, k} / \theta_{B}\right), k=1, \ldots, N$, intercepting the vertical line $\theta_{B}=\theta_{B}^{\prime}$, minus $2 N$ times $\hat{d}$ (which is the intersection of $\theta_{B}=\theta_{B}^{\prime}$ with either $\min _{1 \leq k \leq N}\left(T_{2, k} / \theta_{B}-T_{1, k}\right)$ or $\min _{1 \leq k \leq N}\left(T_{4, k}-T_{3, k} / \theta_{B}\right)$ as proved in Lemma 1 below). Utilizing the fact that $\xi$ depends on two parameters, $\theta_{B}$ and $d$, we will now derive the MLE with the help of the following four lemmas.

Lemma 1: The MLE $\hat{d}$ lies on either $\min _{1 \leq k \leq N}\left(T_{2, k} / \theta_{B}-\right.$ $\left.T_{1, k}\right)$ or $\min _{1 \leq k \leq N}\left(T_{4, k}-T_{3, k} / \theta_{B}\right)$, i.e., on the boundary of the support region.

Proof: See the Appendix.

Lemma 2: The MLE $\hat{d}$ lies either on the uppermost vertex formed by the intersection of the curves $\min _{1 \leq k \leq N}\left(T_{2, k} / \theta_{B}-\right.$ $\left.T_{1, k}\right)$ and $\min _{1 \leq k \leq N}\left(T_{4, k}-T_{3, k} / \theta_{B}\right)$ (shown as point $\mathrm{A}$ in 


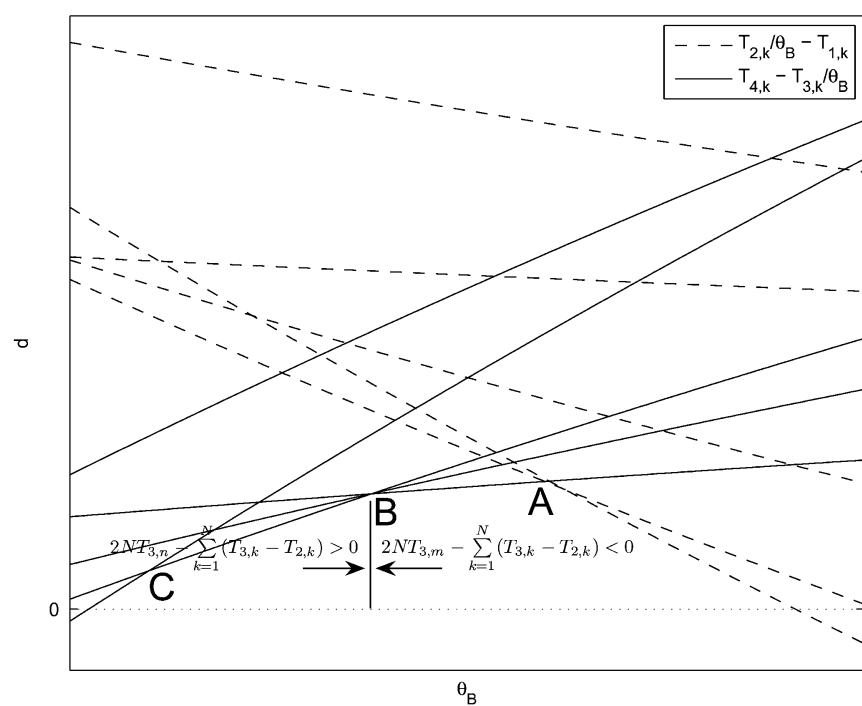

Fig. 4. Zoomed in version of the support region of the likelihood function.

Fig. 4) or on one of the vertices formed by the intersection of the curves $\left(T_{4, k}-T_{3, k} / \theta_{B}\right), k=1, \ldots, N$ (shown as points B, C, etc., in Fig. 4).

Proof: The MLE $\left(\hat{d}, \hat{\theta}_{B}\right)$ is either

$$
\begin{aligned}
\hat{d} & =\frac{T_{2, i} T_{4, j}-T_{1, i} T_{3, j}}{T_{2, i}+T_{3, j}} \\
\hat{\theta}_{B} & =\frac{T_{2, i}+T_{3, j}}{T_{1, i}+T_{4, j}}
\end{aligned}
$$

where the $i, j$ represent the indexes of $\min _{1 \leq k \leq N}\left(T_{2, k} / \theta_{B}-\right.$ $\left.T_{1, k}\right)$ and $\min _{1 \leq k \leq N}\left(T_{4, k}-T_{3, k} / \theta_{B}\right)$, respectively, intersecting at the maximum $\hat{d}$ (which is the uppermost vertex shown as point A in Fig. 4), or

$$
\begin{aligned}
\hat{d} & =T_{4, m}-\frac{T_{3, m}\left(T_{4, m}-T_{4, n}\right)}{T_{3, m}-T_{3, n}} \\
\hat{\theta}_{B} & =\frac{T_{3, m}-T_{3, n}}{T_{4, m}-T_{4, n}} .
\end{aligned}
$$

For proof, see the Appendix.

\section{Algorithm 2: Finding $\hat{\theta}_{B}$ and $\hat{d}$ for $d$ unknown, $\theta_{A}$ known}

1: Find $d^{k, l}=\left(T_{2, k} T_{4, l}-T_{1, k} T_{3, l}\right) /\left(T_{2, k}+T_{3, l}\right)$; $\theta_{B}^{k, l}=\left(T_{2, k}+T_{3, l}\right) /\left(T_{1, k}+T_{4, l}\right) ; \forall k=1, \ldots, N$ and $\forall l=1, \ldots, N$.

2: $(i, j)=\arg \min _{k, l}\left\{d^{k, l}\right\}$;

3: if $2 N T_{3, j}>\sum_{k=1}^{N}\left(T_{3, k}-T_{2, k}\right)$ then

4: $\hat{d}=d^{i, j} ; \hat{\theta}_{B}=\theta_{B}^{i, j}$;

5: else

6: $\quad k=j$;

\section{LABEL:}

7: $\quad$ Find $d^{k, l}=T_{4, k}-\left(T_{3, k}\left(T_{4, k}-T_{4, l}\right)\right) /\left(T_{3, k}-T_{3, l}\right)$; $\theta_{B}^{k, l}=\left(T_{3, k}-T_{3, l}\right) /\left(T_{4, k}-T_{4, l}\right) ; \forall l=1, \ldots, N$

8: $\quad m=k ; n=\arg \max _{l}\left\{\theta_{B}^{k, l} \mid \theta_{B}^{k, l}<\theta_{B}^{i, j}\right\}$;

9: $\quad$ if $2 N T_{3, n}>\sum_{k=1}^{N}\left(T_{3, k}-T_{2, k}\right)$ then

10: $\hat{d}=d^{m, n} ; \hat{\theta}_{B}=\theta_{B}^{m, n}$;
11: else

12: $\quad k=n$;

13: goto LABEL;

14: end if

15: end if

Lemma 3: To the left of the point where $\min _{1 \leq k \leq N}\left(T_{2, k} / \theta_{B}-T_{1, k}\right)$ and $\min _{1 \leq k \leq N}\left(T_{4, k}-T_{3, k} / \theta_{B}\right)$ intersect (i.e., point $\mathrm{A}$ shown in Fig. 4), the boundary of the support region is formed by the curves $\left(T_{4, k}-T_{3, k} / \theta_{B}\right), k=1, \ldots, N$ in such a way that as $\theta_{B}$ increases, a curve $\left(T_{4, m}-T_{3, m} / \theta_{B}\right)$ forms the new boundary of the support region after intersecting the curve $\left(T_{4, n}-T_{3, n} / \theta_{B}\right)$ if and only if $m<n$.

Proof: See the Appendix.

Lemma 4: The MLE $\left(\hat{d}, \hat{\theta}_{B}\right)$, whether (23) and (24) or (28) and (29), is unique.

Proof: See Appendix.

It should be noted that under the most likely scenario, when Node $B$ is sending its timestamps to Node $A$ after short delays, MLE will be given by (23) and (24), but in the usually unlikely scenario of Node $B$ waiting a long period of time before sending one of its timestamps to Node A, (28) and (29) can be the MLE only if $2 N T_{3, j}<\sum_{k=1}^{N}\left(T_{3, k}-T_{2, k}\right)$. Note that in this case, in addition to previous $N^{2}$ intersections, $N-1$ more intersections have to be compared for each $j$ satisfying $2 N T_{3, j}<\sum_{k=1}^{N}\left(T_{3, k}-T_{2, k}\right)$. The whole procedure for finding this MLE is summarized in Algorithm 2. This algorithm proceeds in precisely the same steps as described above.

Now that we have obtained some insight into this problem for $\theta_{A}$ known, we next proceed with the situation when $\theta_{A}$ is unknown.

\section{Case III: d Known, $\theta_{A}$ Unknown}

The likelihood function in this case is the same as (3), where $d$ is fixed and known. The region over which the likelihood function is nonzero is given by indicator function $I[\cdot]$ in (3) and shown in Fig. 5. This 3-D support region is dramatically more complex than what we observed in the first two cases. It is also evident from (3) that $\xi$ is the same as in previous cases and the likelihood function can again be maximized by minimizing $\xi$. Since $\sum_{k=1}^{N}\left(T_{2, k}-T_{3, k}\right)$ is always negative and $d$ is given, $\xi$ can be minimized by taking $\theta_{B}$ as small as possible. To find this minimum $\theta_{B}$, we take a horizontal slice from this 3-D support region at the constant $d$. This gives an aerial view of the 2-D region shown in Fig. 6 highlighting the relation between $\theta_{B}$ versus $\theta_{A}$ for the known $d$. Therefore, in accordance with (3), we can express the support of the likelihood function in the form of the following constraints:

$$
\begin{aligned}
-\infty & <\theta_{A}<\infty \\
\frac{T_{3, k}-\theta_{A}}{T_{4, k}-d} \leq \theta_{B} & \leq \frac{T_{2, k}-\theta_{A}}{T_{1, k}+d} ; \quad k=1, \ldots, N .
\end{aligned}
$$

These constraints can be viewed as $\theta_{B}$ being a monotonically decreasing function of $\theta_{A} \forall k$ due to the positivity of $\left(T_{1, k}+d\right)$ and $\left(T_{4, k}-d\right)$, and the shaded region is the one which satisfies these constraints. 


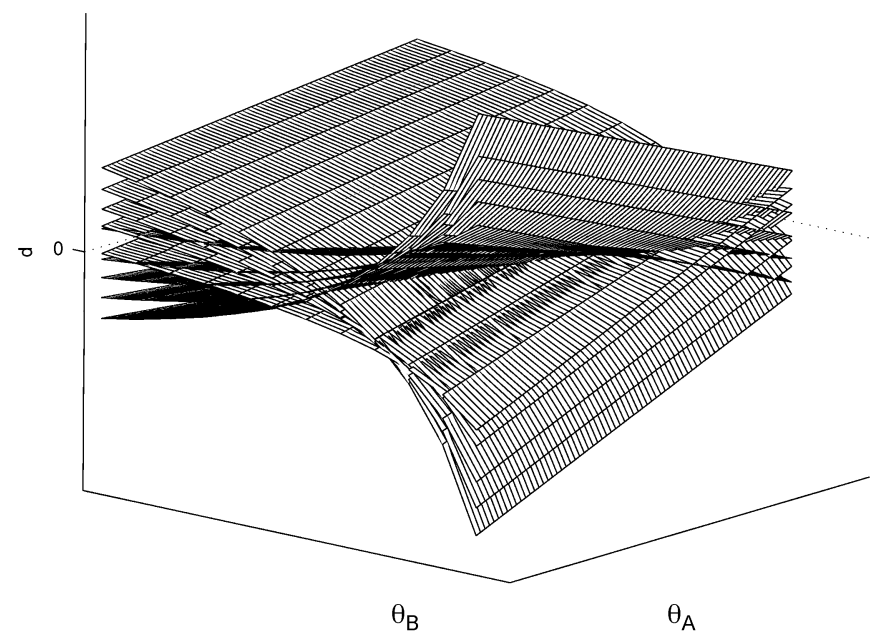

Fig. 5. $d$ as a function of $\theta_{A}$ and $\theta_{B}$.

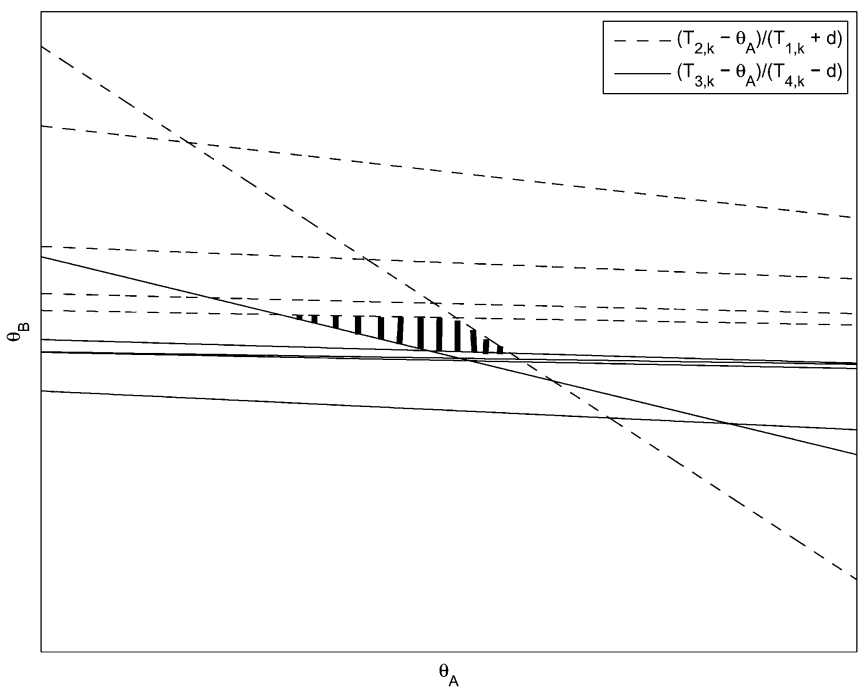

Fig. 6. $\theta_{B}$ as a function of $\theta_{A}$ for constant $d$.

Lemma 5: Of all the intersections of $\left(T_{2, k}-\theta_{A}\right) /\left(T_{1, k}+d\right)$ with $\left(T_{3, k}-\theta_{A}\right) /\left(T_{4, k}-d\right)$, only two points satisfy the constraints (11) and (12) in a way that they represent the starting and ending points of the support region and the point with minimum $\theta_{B}$ is the one with maximum $\theta_{A}$.

Proof: See the Appendix.

We can minimize $\xi$ by taking the intersection of $\min _{1 \leq k \leq N}\left(T_{2, k}-\theta_{A}\right) /\left(T_{1, k}+d\right)$ and $\max _{1 \leq k \leq N}\left(T_{3, l}-\right.$ $\left.\theta_{A}\right) /\left(T_{4, l}-d\right)$ at minimum possible $\hat{\theta}_{B}$, which gives the MLE $\left(\hat{\theta}_{A}, \hat{\theta}_{B}\right)$ as

$$
\begin{aligned}
\hat{\theta}_{A} & =\frac{T_{3, j}\left(T_{1, i}+d\right)-T_{2, i}\left(T_{4, j}-d\right)}{\left(T_{1, i}+d\right)-\left(T_{4, j}-d\right)} \\
\hat{\theta}_{B} & =\frac{T_{2, i}-T_{3, j}}{\left(T_{1, i}+d\right)-\left(T_{4, j}-d\right)}
\end{aligned}
$$

where the indexes $(i, j)$ are the ones whose intersection gives the minimum allowed $\hat{\theta}_{B}$. Algorithm 3 presents in detail the steps that are required for finding this MLE.

Algorithm 3 first finds all the intersections and chooses two candidate points $\left(\theta_{A}^{\prime}, \theta_{B}^{\prime}\right)_{1}$ and $\left(\theta_{A}^{\prime}, \theta_{B}^{\prime}\right)_{2}$ such that $\theta_{B}^{\prime} \leq$
$\left(T_{2, k}-\theta_{A}^{\prime}\right) /\left(T_{1, k}+d\right) \forall k$ and $\theta_{B}^{\prime} \geq\left(T_{3, l}-\theta_{A}^{\prime}\right)\left(T_{4, l}-d\right) \forall l$. These are the starting and ending points of the nonzero likelihood region as proved in Lemma 5 above and the point with minimum $\theta_{B}$ (which corresponds to the one with maximum $\left.\theta_{A}\right)$ is chosen.

\section{Algorithm 3: Finding $\hat{\theta}_{A}$ and $\hat{\theta}_{B}$ for $d$ known, $\theta_{A}$ unknown}

1: Find $\theta_{A}^{k, l}=\left(T_{3, l}\left(T_{1, k}+d\right)-T_{2, k}\left(T_{4, l}-d\right)\right) /\left(\left(T_{1, k}+d\right)\right.$ $\left.-\left(T_{4, l}-d\right)\right) ; \theta_{B}^{k, l}=\left(T_{2, k}-T_{3, l}\right) /\left(\left(T_{1, k}+d\right)-\left(T_{4, l}-d\right)\right)$; $\forall k=1, \ldots, N$ and $\forall l=1, \ldots, N$;

2: $(i, j)=\left\{(k, l) \mid \theta_{B}^{k, l} \leq\left(T_{2, r}-\theta_{A}^{k, l}\right) /\left(T_{1, r}+d\right) \forall r\right.$ and $\left.\theta_{B}^{k, l} \geq\left(T_{3, r}-\theta_{A}^{k, l}\right) /\left(T_{4, r}-d\right) \forall r\right\}$

3: $(m, n)=\left\{(k, l) \mid(k, l) \neq(i, j), \theta_{B}^{k, l} \leq\left(T_{2, r}-\theta_{A}^{k, l}\right) /\right.$ $\left(T_{1, r}+d\right) \forall r$ and $\left.\theta_{B}^{k, l} \geq\left(T_{3, r}-\theta_{A}^{k, l}\right) /\left(T_{4, r}-d\right) \forall r\right\} ;$

4: $\hat{\theta}_{B}=\min \left\{\theta_{B}^{i, j}, \theta_{B}^{m, n}\right\}$;

5: $\hat{\theta}_{A}=\max \left\{\theta_{A}^{i, j}, \theta_{A}^{m, n}\right\}$;

\section{Case IV: d Unknown, $\theta_{A}$ Unknown}

In this case, all of $d, \theta_{A}$ and $\theta_{B}$ are unknown and have to be jointly estimated. The likelihood function in this case is the same as in (3) but $d$ is unknown. The region where the likelihood function is nonzero can be expressed in the form of the following constraints:

$$
\begin{aligned}
-\infty & <\theta_{A}<\infty, d>0, \theta_{B}>0 \\
d & \leq \frac{T_{2, k}-\theta_{A}}{\theta_{B}}-T_{1, k}, \quad k=1, \ldots, N \\
d & \leq T_{4, k}-\frac{T_{3, k}-\theta_{A}}{\theta_{B}}, \quad k=1, \ldots, N .
\end{aligned}
$$

Within the constraint $d>0,\left(T_{2, k}-\theta_{A}\right) / \theta_{B}-T_{1, k}$ are monotonically decreasing functions of $\theta_{A}$ and $\theta_{B} \forall k$, and $T_{4, k}-$ $\left(T_{3, k}-\theta_{A}\right) / \theta_{B}$ are monotonically increasing functions of $\theta_{A}$ and $\theta_{B} \forall k$ as shown in Fig. 5. It is clear from the same figure that the nonzero likelihood region is similar in shape to a dome if we look at it standing on $\left(\theta_{A}, \theta_{B}\right)$ plane. Lemma 1 asserts that the MLE $\left(\hat{d}, \hat{\theta}_{A}, \hat{\theta}_{B}\right)$ should lie somewhere on the ceiling of this dome. The lines on $\left(\theta_{A}, \theta_{B}\right)$ plane, on which the intersections of the surfaces lie are given by

$$
\begin{array}{r}
\theta_{A}=\frac{1}{2}\left[\left(T_{2, k}+T_{3, l}\right)-\theta_{B}\left(T_{1, k}+T_{4, l}\right)\right], \\
k=1, \ldots, N ; \quad l=1, \ldots, N
\end{array}
$$

or equivalently

$$
\theta_{B}=\frac{T_{2, k}+T_{3, l}-2 \theta_{A}}{T_{1, k}+T_{4, l}}, \quad k=1, \ldots, N ; l=1, \ldots, N .
$$

Note that putting $\theta_{B}=1$ (the case when there is no clock skew) in (15) and taking the minimum results in the MLE $\hat{\theta}_{A}$ in (4) derived by [2]. Although $d$ is a function of both $\theta_{A}$ and $\theta_{B}$, it can be written as a function of either $\theta_{A}$ only or $\theta_{B}$ only by utilizing this linear relationship between these two parameters. Fig. 7 shows the imaginary 2-D region where $d$ is drawn as a function of $\theta_{B}$ only and Fig. 8 shows the imaginary 2-D region where $d$ is drawn as a function of $\theta_{A}$ only. Note that 


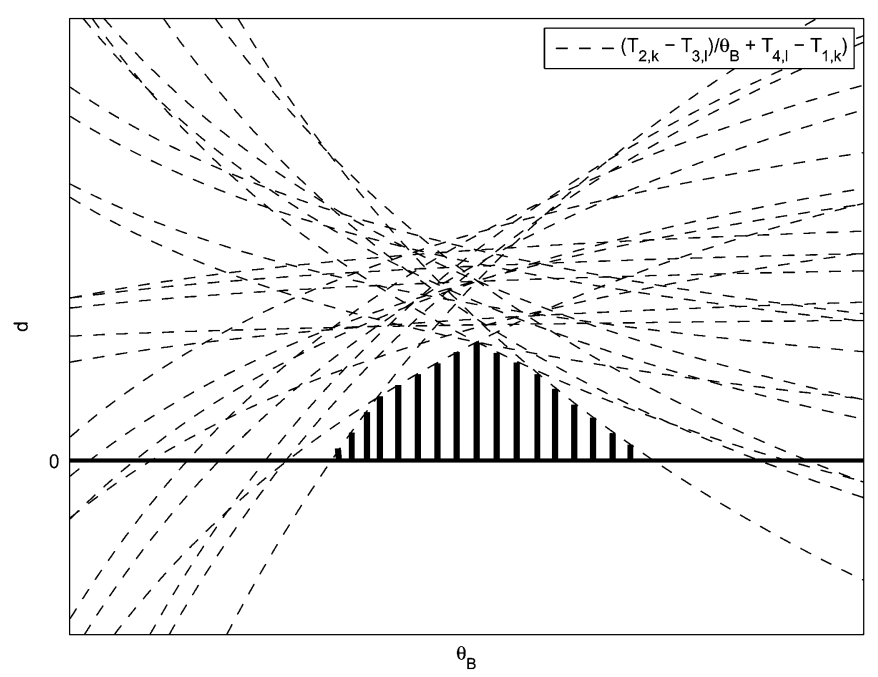

Fig. 7. $d$ as a function of $\theta_{B}$ only.

these are actually 3-D plots, but the points on the bottom two axes $\left(\theta_{A}, \theta_{B}\right)$ are replaced with $\left(\frac{1}{2}\left[\left(T_{2, k}+T_{3, l}\right)-\theta_{B}\left(T_{1, k}+\right.\right.\right.$ $\left.\left.\left.T_{4, l}\right)\right], \theta_{B}\right)$ and $\left(\theta_{A},\left(T_{2, k}+T_{3, l}-2 \theta_{A}\right) /\left(T_{1, k}+T_{4, l}\right)\right)$ in Figs. 7 and 8 , respectively.

Over the line (15), $d$ is given by

$$
\begin{aligned}
& d \leq \frac{1}{2}\left[\frac{T_{2, k}-T_{3, l}}{\theta_{B}}+\left(T_{4, l}-T_{1, k}\right)\right], \\
& k=1, \ldots, N ; l=1, \ldots, N .
\end{aligned}
$$

Note that putting $\theta_{B}=1$ and taking the min results in MLE $\hat{d}$ given by [2]. And over the line (16), $d$ is given by

$$
\begin{array}{r}
d \leq \frac{T_{2, k} T_{4, l}-T_{1, k} T_{3, l}+\theta_{A}\left(T_{1, k}-T_{4, l}\right)}{T_{2, k}+T_{3, l}-2 \theta_{A}}, \\
k=1, \ldots, N ; l=1, \ldots, N .
\end{array}
$$

A closer look at (18) reveals that its RHS goes to $-\infty$ or $+\infty$ respectively at $\theta_{A}=\left(T_{2, i}+T_{3, j}\right) / 2$ according to the negative or positive sign of the numerator. But the constraint $d>0$ automatically restricts the nonzero likelihood region well before even the first discontinuity of this kind as shown in Fig. 8.

1) Estimating $d$ and $\theta_{B}$ : Consider the set of $N^{2}$ curves given in (17) and plotted in Fig. 7. Since the signs of $T_{2, k}-T_{3, l}$ and $T_{4, l}-T_{1, k}$ are always opposite, $N(N-1) / 2$ of these curves have positive numerator in the term involving $\theta_{B}$ and negative constant term, while the remaining $N(N+1) / 2$ have negative numerator in the term involving $\theta_{B}$ and positive constant term. Based on this observation, (17) can be written in the form of two sets of inequalities such that $\left(T_{2, k}-T_{3, l}\right)>0$ for one set and $\left(T_{2, k}-T_{3, l}\right)<0$ for the other as shown in Fig. 7. Then the current scenario assumes quite a similar form to the set of constraints (8) and (9). Therefore, initially a total of $[N(N-$ 1)/2][N(N+1)/2] $=N^{2}\left(N^{2}-1\right) / 4$ intersections (denoted by $d^{k, l, m, n}$ in Algorithm 4) are to be compared. Lemmas 1,2, 3 , and 4 are then similarly true for these sets of inequalities and the MLEs can be derived by following a similar procedure. Let us denote $\left\{\min _{1 \leq k, l \leq N}(1) /(2)\left[\left(T_{2, k}-T_{3, l}\right) / \theta_{B}+\left(T_{4, l}-\right.\right.\right.$ $\left.\left.\left.T_{1, k}\right)\right] \mid\left(T_{2, k}-T_{3, l}\right)>0\right\}$ as $\left(T_{2, i}-T_{3, j}\right) / 2 \theta_{B}+\left(T_{4, j}-\right.$

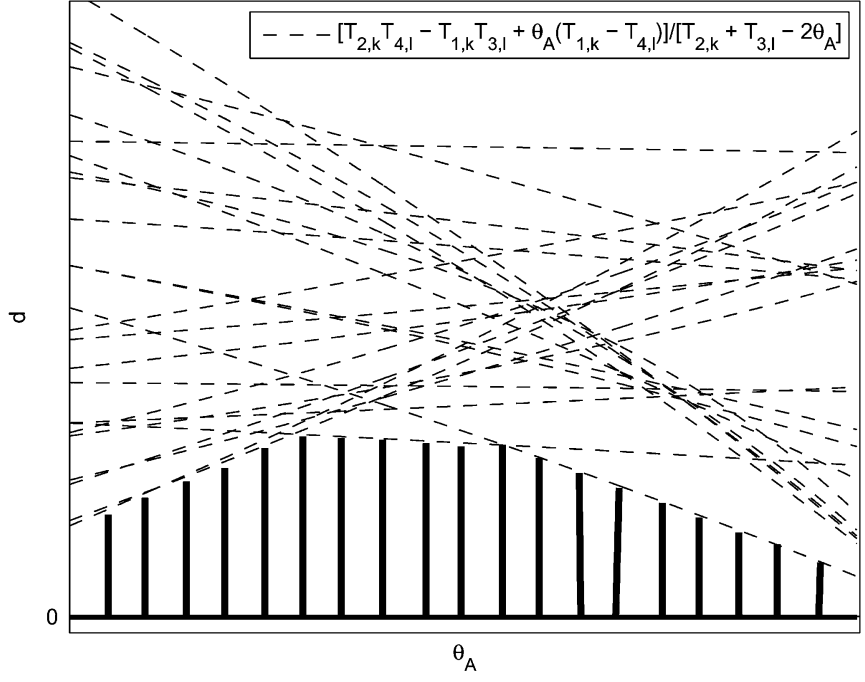

Fig. 8. $d$ as a function of $\theta_{A}$ only.

$\left.T_{1, i}\right) / 2$ and $\left\{\min _{1 \leq k, l \leq N}(1) /(2)\left[\left(T_{2, k}-T_{3, l}\right) / \theta_{B}+\left(T_{4, l}\right.\right.\right.$ $\left.\left.\left.T_{1, k}\right)\right] \mid\left(T_{2, k}-T_{3, l}\right)<0\right\}$ as $\left(T_{2, m}-T_{3, n}\right) / 2 \theta_{B}+\left(T_{4, n}-\right.$ $\left.T_{1, m}\right) / 2$. Then if $\sum_{k=1}^{N}\left[T_{2, k}-T_{3, k}-\left(T_{2, m}-T_{3, n}\right)\right]$ is positive, the MLE $\left(\hat{d}, \hat{\theta}_{B}\right)$ is the intersection of this curve with the one discussed above, i.e.,

$$
\begin{aligned}
\hat{d}=\frac{1}{2}[ & \frac{\left(T_{2, i}-T_{3, j}\right)\left(\left(T_{1, i}-T_{1, m}\right)+\left(T_{4, n}-T_{4, j}\right)\right)}{\left(T_{2, i}-T_{2, m}\right)+\left(T_{3, n}-T_{3, j}\right)} \\
& \left.+\left(T_{4, j}-T_{1, i}\right)\right]
\end{aligned}
$$

and

$$
\hat{\theta}_{B}=\frac{\left(T_{2, i}-T_{2, m}\right)+\left(T_{3, n}-T_{3, j}\right)}{\left(T_{1, i}-T_{1, m}\right)+\left(T_{4, n}-T_{4, j}\right)} .
$$

Otherwise, if $\sum_{k=1}^{N}\left[T_{2, k}-T_{3, k}-\left(T_{2, m}-T_{3, n}\right)\right]$ is negative, then the MLE is the intersection of the curves $\left(T_{2, p}-T_{3, q}\right) / 2 \theta_{B}+\left(T_{4, q}-T_{1, p}\right) / 2$ and $\left(T_{2, r}-T_{3, s}\right) / 2 \theta_{B}+$ $\left(T_{4, s}-T_{1, r}\right) / 2$ (denoting the intersections of the curves in (17) as $d^{k, l, m, n}, \forall(k, l, m, n)$, and $d^{k, l, m, n}$ satisfy the constraints (13) and (14)), where

$$
\begin{aligned}
(p, q, r, s)= & \arg \max _{k, l, m, n}\left\{d^{k, l, m, n} \mid N\left(T_{3, l}-T_{2, k}\right)\right. \\
& <\sum_{k=1}^{N}\left(T_{3, k}-T_{2, k}\right) ; \\
& \left.N\left(T_{3, n}-T_{2, m}\right)>\sum_{k=1}^{N}\left(T_{3, k}-T_{2, k}\right)\right\} .
\end{aligned}
$$

Hence, here the MLE $\left(\hat{d}, \hat{\theta}_{B}\right)$ is

$$
\begin{aligned}
\hat{d}=\frac{1}{2}[ & \frac{\left(T_{2, p}-T_{3, q}\right)\left(\left(T_{1, p}-T_{1, r}\right)+\left(T_{4, s}-T_{4, q}\right)\right)}{\left(T_{2, p}-T_{2, r}\right)+\left(T_{3, s}-T_{3, q}\right)} \\
& \left.+\left(T_{4, q}-T_{1, p}\right)\right]
\end{aligned}
$$

and

$$
\hat{\theta}_{B}=\frac{\left(T_{2, p}-T_{2, r}\right)+\left(T_{3, s}-T_{3, q}\right)}{\left(T_{1, p}-T_{1, r}\right)+\left(T_{4, s}-T_{4, q}\right)} .
$$




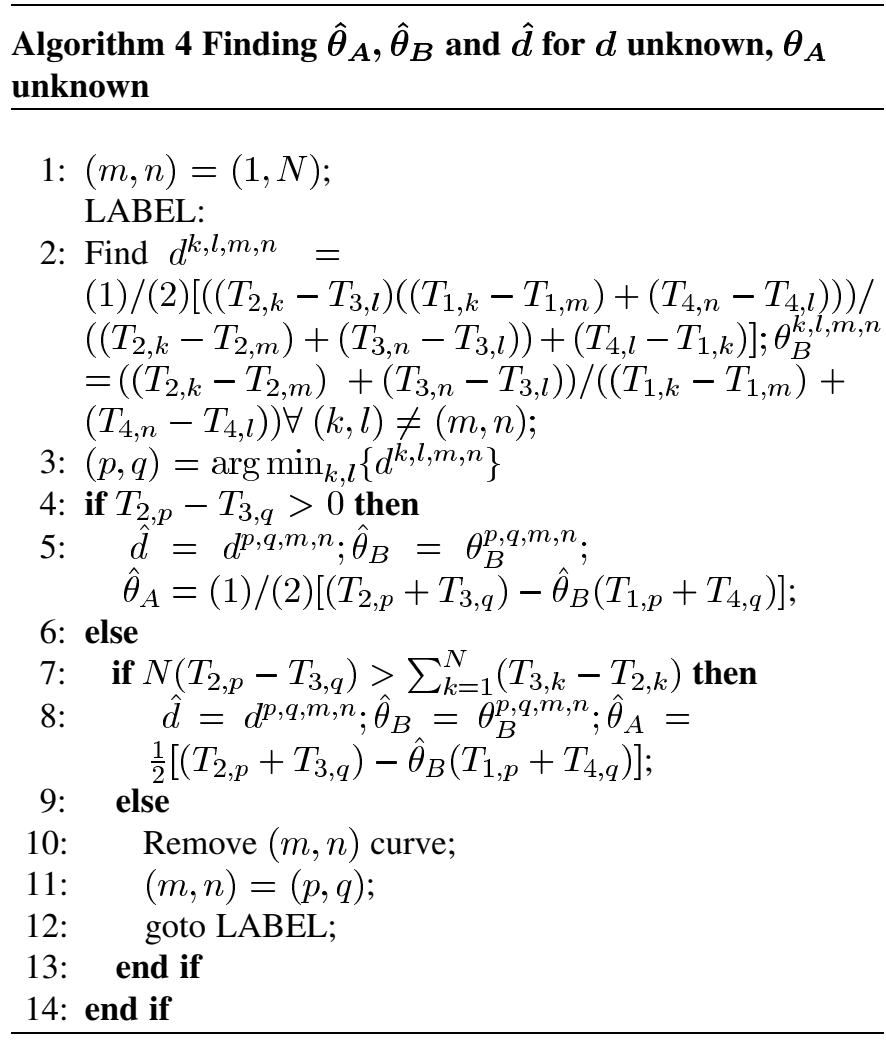

The complete procedure for finding the MLE is described in Algorithm 4. Although a modified Algorithm 2 can be used in this case, we present this alternative algorithm for the sake of completion. It starts from the curve for which $\left(T_{2, m}-T_{3, n}\right)$ is minimum, i.e., $\left(T_{2,1}-T_{3, N}\right)$ and then compares its intersections with other curves. It keeps on replacing this curve with the one giving the next minimum $d^{k, l, m, n}$ within the constraints until the MLE is found according to the procedure described before.

2) Estimating $\theta_{A}$ : A simpler and easier to implement method is estimating $\hat{\theta}_{A}$ by noting that for every $d$ as a function of $\theta_{B}$ (and hence the one minimizing $\xi$ ), there is a corresponding $\theta_{A}$ according to (15). Therefore, the MLE is

$$
\begin{aligned}
& \hat{\theta}_{A}=\frac{1}{2}\left[\left(T_{2, i}+T_{3, j}\right)-\hat{\theta}_{B}\left(T_{1, i}+T_{4, j}\right)\right] \\
& \hat{\theta}_{A}=\frac{1}{2}\left[\left(T_{2, p}+T_{3, q}\right)-\hat{\theta}_{B}\left(T_{1, p}+T_{4, q}\right)\right]
\end{aligned}
$$

depending on whether $\hat{\theta}_{B}$ is given by (19) or (20). The reason for not following the same procedure as in finding $\hat{\theta}_{B}$ by using (16) is that the problem becomes computationally complex. First, the likelihood function assumes quite a complicated form after plugging (16) and (18) into (3). Second, the intersection $\hat{\theta}_{A}$ of the curves in (16) has to be found by solving quadratic equations with large coefficients. To be exact, $\hat{\theta}_{A}$ is the solution of

$$
\begin{aligned}
2 \hat{\theta}_{A}^{2}[ & \left.\left(T_{1, r}-T_{1, p}\right)+\left(T_{4, s}-T_{4, q}\right)\right] \\
+ & \hat{\theta}_{A}\left[\left(T_{1, p}-T_{4, q}\right)\left(T_{2, r}+T_{3, s}\right)\right. \\
& \left.\quad-\left(T_{1, r}-T_{4, s}\right)\left(T_{2, p}+T_{3, q}\right)\right] \\
+ & {\left[T_{2, p} T_{2, r}\left(T_{4, q}-T_{4, s}\right)+T_{2, p} T_{3, s}\left(T_{4, q}+T_{1, r}\right)\right.} \\
& \left.\quad-T_{2, r} T_{3, q}\left(T_{4, s}+T_{1, p}\right)-T_{3, q} T_{3, s}\left(T_{1, r}-T_{1, p}\right)\right]=0
\end{aligned}
$$

where the indexes $p, q, r, s$ are the ones minimizing $\xi$. It has two solutions and the solution which gives

$$
\hat{\theta}_{A}<\min \left(T_{2, i}+T_{3, j}\right) / 2, i=1, \ldots, N ; j=1, \ldots, N
$$

is accepted to satisfy the constraints set by $I[\cdot]$ in (3). Hence, (21) or (22) should be chosen to estimate $\theta_{A}$ on the grounds of lesser computational complexity. It should be noted that $\hat{d}$ will be the same in both approaches when we estimate it jointly with $\hat{\theta}_{B}$ and $\hat{\theta}_{A}$ whether by expressing it in terms of $\theta_{B}$ only or in terms of $\theta_{A}$ only. Algorithm 4 also includes the step for estimating $\theta_{A}$.

\section{Proposed Algorithm}

Although a little complex, the MLE can be implemented in a WSN to achieve clock synchronization. However, simpler algorithms, even with the sacrifice of some performance degradation, are more suited to the WSN requirements. In this section, we present an easier to implement algorithm which requires less number of computations at the expense of increased mean square error (MSE). The intuition behind the idea is that (1) and (2) can be rewritten as

$$
\begin{aligned}
& T_{2, k}=T_{1, k} \theta_{B}+\theta_{A}+\left(d+X_{k}\right) \theta_{B} \\
& T_{3, k}=T_{4, k} \theta_{B}+\theta_{A}-\left(d+Y_{k}\right) \theta_{B} .
\end{aligned}
$$

Notice that since $\theta_{B}, d, X_{k}$ and $Y_{k}$ are all positive, the points $T_{2, k}, k=1, \ldots, N$ will always be above the line $T_{1, k} \theta_{B}+\theta_{A}$ and the points $T_{3, k}, k=1, \ldots, N$ will always be below the line $T_{4, k} \theta_{B}+\theta_{A}$. Hence, a good estimate of $\theta_{B}$ and $\theta_{A}$ can be formed by fitting a line between the observations such that $T_{2, k}, k=1, \ldots, N$ are above the fitted line and $T_{3, k}, k=$ $1, \ldots, N$ are below it. The strategy we have devised for a good estimate is to join the two points $P_{1}$ and $P_{2}$, where $P_{1}$ corresponds to $(1) /(2) \min _{1 \leq k \leq N}\left\{T_{4, k}-T_{1, k}\right\}$ and $P_{2}$ corresponds to $(1) /(2) \min _{1 \leq k \leq N, k \neq i}\left\{T_{4, k}-T_{1, k}\right\}$. Representing their indexes by $i$ and $j$, respectively, we have

and

$$
P_{1}=\left\{\frac{1}{2}\left(T_{4, i}-T_{1, i}\right), \frac{1}{2}\left(T_{2, i}+T_{3, i}\right)\right\}
$$

$$
P_{2}=\left\{\frac{1}{2}\left(T_{4, j}-T_{1, j}\right), \frac{1}{2}\left(T_{2, j}+T_{3, j}\right)\right\}
$$

i.e., $P_{1}$ and $P_{2}$ correspond to the first two order statistics of the data set $(1 / 2)\left(T_{4, k}-T_{1, k}\right), k=1, \ldots, N$. The line formed by joining those two points is shown in Fig. 9 along with the true curve. Hence, the estimate $\left(\hat{\theta}_{B}, \hat{\theta}_{A}\right)$ can be expressed as

$$
\begin{aligned}
\hat{\theta}_{B} & =\frac{\left(T_{2, i}+T_{3, i}\right) / 2-\left(T_{2, j}+T_{3, j}\right) / 2}{\left(T_{1, i}+T_{4, i}\right) / 2-\left(T_{1, j}+T_{4, j}\right) / 2}, \\
\hat{\theta}_{A} & =\left(T_{2, i}+T_{3, i}\right) / 2-\hat{\theta}_{B}\left(T_{1, i}+T_{4, i}\right) / 2 .
\end{aligned}
$$




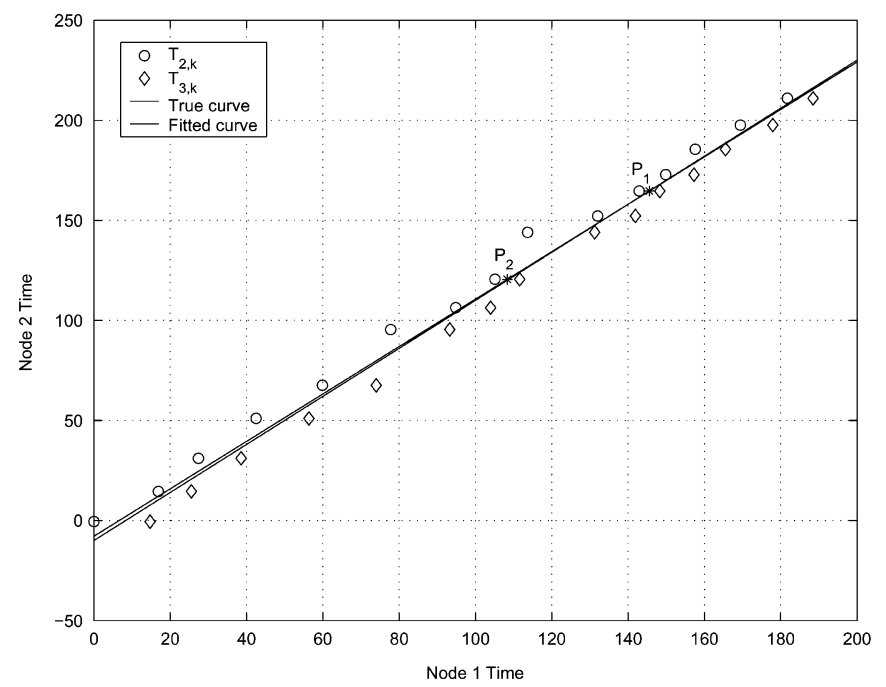

Fig. 9. Estimated fit with the original curve.

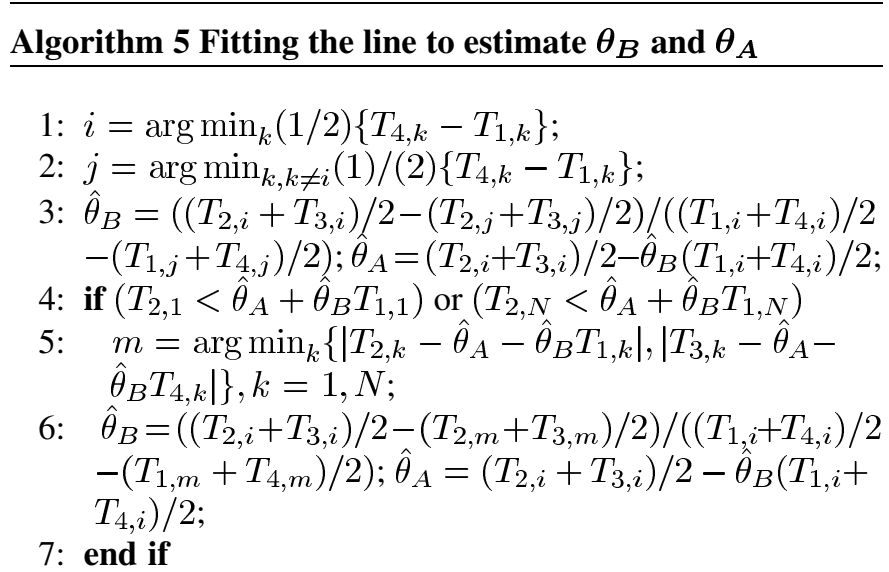

When $P_{1}$ and $P_{2}$ fall very close to each other, it may happen that the fitted line exits from its boundaries and a part of it becomes either greater than some $T_{2, k}$ or less than some $T_{3, k}$. In that case, we propose to join the minimum point $P_{1}$ with one of the boundary points $\left\{T_{2,1}, T_{2, N}, T_{3,1}, T_{3, N}\right\}$ depending on which of them has the shortest distance from the initial fitted line. This algorithm is extremely simple since it just involves finding the first two order statistics from a set of $N$ observations and checking the boundary conditions for the two extreme points. If the fitted line violates the boundary condition, the estimator is again formed by the same simple formula but with a point having different time index. Since this point is on the boundary, the procedure does not have to be repeated and there are no loops involved as before. The whole procedure for finding these estimates is described in Algorithm 5.

Some additional advantages of using Algorithm 5 are that $\theta_{A}$ can also be estimated by the $y$ intercept of the fitted line and importantly, $d$ does not need to be known.

\section{Simulation Results}

We have simulated the performance of the MLE for fixed delay $d=2$, clock offset $\theta_{A}=-10$, exponential delay parameter $\alpha=2$ and for two different clock skews $\theta_{B}=1.0007$ and $\theta_{B}=1.003$. The reason of choosing different clock skews is

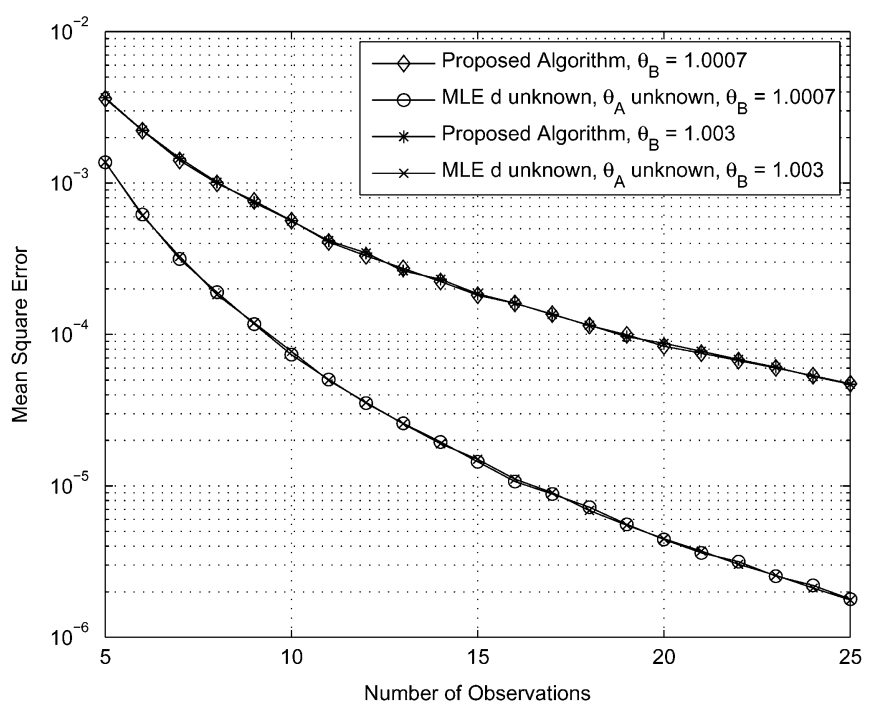

Fig. 10. Comparison of the proposed algorithm with case IV.

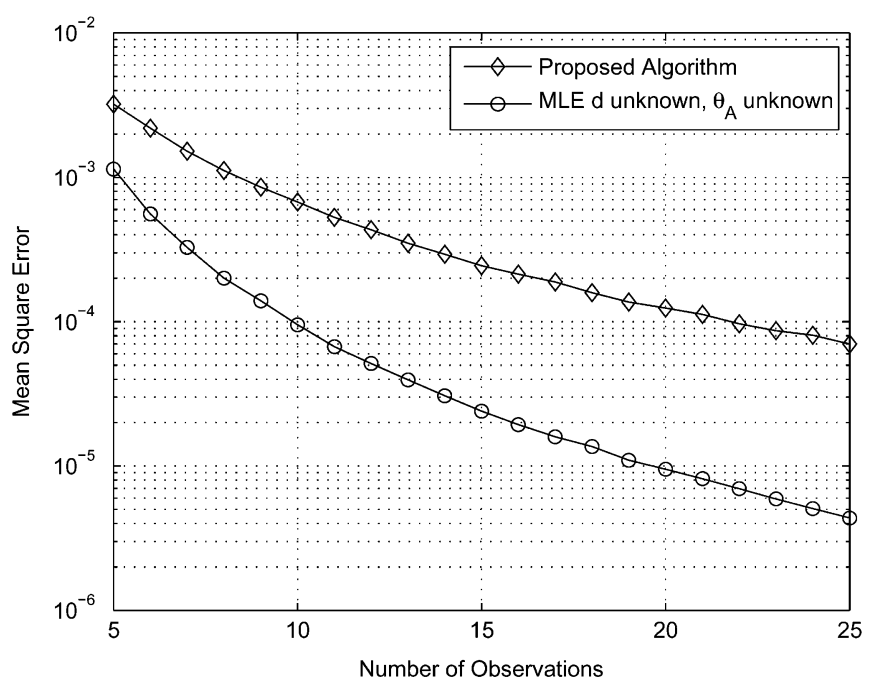

Fig. 11. Comparison of the proposed algorithm with case IV for Gamma distributed random delays.

to show a comparison of these algorithms on the performance for various actual parameters. We compare the performance of our proposed algorithm with the most general (and similar) case when $\left(d, \theta_{A}, \theta_{B}\right)$ have to be jointly estimated. Fig. 10 plots the mean square error of both clock skew estimators for $\theta_{B}=1.0007$ and $\theta_{B}=1.003$ against the number of message exchanges. It is clear from Fig. 10 that although the MLE performs better than the proposed algorithm, it can still be adopted with the sacrifice of some performance in the scenarios where energy conservation is the main issue of concern. Hence, in the light of the accuracy energy tradeoff for attaining such a gain in performance by deploying MLE, we assert that the proposed algorithm is very suitable for WSNs. Moreover, there is not any significant difference between the mean square error of the MLE and that of the proposed algorithm for different set of actual parameters and hence it is suited to different types of sensor nodes used today.

To check the robustness of our proposed algorithm against possible model mismatches, we have plotted the performance of the MLE in the most general Case IV and our proposed algorithm in Fig. 11 when the actual random delays come from the 
TABLE I

COMPUTATIONAL COMPLEXITY OF EACH ALGORITHM

\begin{tabular}{|c|c|c|c|}
\hline & Additions & Multiplications & Divisions \\
\hline Algorithm 1 & $2 N-1$ & 0 & $N$ \\
\hline Algorithm 2 (1 cycle) & $5 N^{2}+5 N$ & $2 N^{2}+4$ & $2 N^{2}+2 N$ \\
\hline Algorithm 3 & $4 N^{3}+5 N^{2}+2$ & $2 N^{2}$ & $2 N^{3}+2 N^{2}$ \\
\hline Algorithm 4 (1 cycle) & $9 N^{2}+N+6$ & $2 N^{2}+5$ & $2 N^{2}$ \\
\hline Proposed Algorithm & $3 N+31$ & $N+10$ & 2 \\
\hline
\end{tabular}

widely used Gamma distribution instead of the exponential distribution. Fig. 11 shows the mean square error of both of these algorithms against the number of observations when the random delays were simulated as Gamma random variables with shape parameter 2 and scale parameter 1 . It is interesting to observe that the difference between their performance still remains on the same scale as in Fig. 10. Therefore, the proposed algorithm is not only computationally simple and easy to implement but also robust to different environments.

\section{COMPUTATIONAL COMPLEXITY COMPARISON}

Table I presents the number of operations required for all the algorithms. Note that these numbers have been calculated by considering the necessary simplifications (e.g., storing the output of an operation if it is to be used later). In addition, the operation count for Algorithm 2 and Algorithm 4 is given assuming no jumps. When their respective conditional statements become true, the code will jump around in the loop and the operation count will be multiplied by the number of jumps. Moreover, it must be kept in mind that the division is the most complex algorithm to implement in a DSP and the number of division operations must be given the highest weight while choosing between different algorithms. Finally, the operation count of our proposed algorithm is given for the worst case scenario, the probability of which is very low. For usual operation, its complexity will only be $3 N+11$ additions, $N+4$ multiplications and 1 division.

For a comparison, observe that even for a small number of observations, e.g., 10, Algorithm 4 requires 916 additions, 205 multiplications and 200 divisions. On the other hand, the proposed algorithm requires only 61 additions, 20 multiplications and 2 divisions for 10 observations in the worst case. As the number of observations $N$ increases, the difference between their operation counts increases significantly while the difference between their MSE decreases, making it a more viable option for large $N$. However, it must be remembered that in the light of the results by Pottie and Kaiser [21], who have reported that the energy required to transmit 1 bit over 100 meters (3 Joules) is equivalent to the energy required to execute 3 million instructions, employing the MLE to achieve clock synchronization in a WSN is still a practical option.

\section{CONCLUSION AND FUtURE WORK}

The maximum likelihood estimators of both the clock offset and skew for any general time synchronization protocol involving a two way message exchange mechanism are derived assuming exponential delays. The complete algorithms used for finding these MLEs are also presented. In addition, due to the complexity of the MLEs, a simple algorithm is proposed which takes much lesser number of computations at the cost of some degradation in quality. For future, we plan to extend this methodology to analyze other time synchronization protocols for both single hop and multihop cases. Also, finding the Cramér-Rao lower bounds (CRLBs) for the clock offset and skew estimators derived here represents an important open research problem. However, it must be noted that the CRLB in this case can not be derived by the same procedure as in [14]. The reason is that $\min _{1 \leq k \leq N}\left(T_{2, k} / \theta_{B}-T_{1, k}\right)$ and $\min _{1 \leq k \leq N}\left(T_{4, k}-T_{3, k} / \theta_{B}\right)$ in Case II intersecting at optimal $\left(\hat{d}, \hat{\theta}_{B}\right)$ (and similar curves in other cases) do not correspond to $\min _{1 \leq k \leq N} X_{k}$ and $\min _{1 \leq k \leq N} Y_{k}$ respectively. In addition, all the order statistics from an exponential distribution, except the first, do not have exponential distribution. Additionally, exploring the effects of violation of i.i.d. assumption for the random delays, missing data points due to communication losses, or quantization errors are interesting open problems.

\section{APPENDIX}

Lemma 1: The MLE $\hat{d}$ lies on either $\min _{1 \leq k \leq N}\left(T_{2, k} / \theta_{B}-\right.$ $\left.T_{1, k}\right)$ or $\min _{1 \leq k \leq N}\left(T_{4, k}-T_{3, k} / \theta_{B}\right)$, i.e., on the boundary of the support region.

Proof: This can be proved by contradiction. Let us assume that the $\hat{d}$ does not lie on the boundary, but somewhere else inside the support region. Then for some minimizing $\hat{\theta}_{B}, \xi$ can be further decreased by increasing $\hat{d}$ to the top of the allowable region (which coincides with one of the above mentioned curves) for the same $\hat{\theta}_{B}$, hence a contradiction.

Lemma 2: The MLE $\hat{d}$ lies either on the uppermost vertex formed by the intersection of the curves $\min _{1 \leq k \leq N}\left(T_{2, k} / \theta_{B}-\right.$ $T_{1, k}$ ) and $\min _{1 \leq k \leq N}\left(T_{4, k}-T_{3, k} / \theta_{B}\right.$ ) (shown as point $\mathrm{A}$ in Fig. 4) or on one of the vertices formed by the intersection of the curves $\left(T_{4, k}-T_{3, k} / \theta_{B}\right), k=1, \ldots, N$ (shown as points B, C, etc., in Fig. 4).

Proof: It is straightforward to notice from (10) that when $T_{2, k}=T_{3, k}$, for all $k, \xi$ can be minimized by making $d$ as large as possible, which is the intersection of the curves $\min _{1 \leq k \leq N}\left(T_{2, k} / \theta_{B}-T_{1, k}\right)$ and $\min _{1 \leq k \leq N}\left(T_{4, k}-T_{3, k} / \theta_{B}\right)$. Hence, the MLE $\left(\hat{d}, \hat{\theta}_{B}\right)$ is

and

$$
\hat{d}=\frac{T_{2, i} T_{4, j}-T_{1, i} T_{3, j}}{T_{2, i}+T_{3, j}}
$$

$$
\hat{\theta}_{B}=\frac{T_{2, i}+T_{3, j}}{T_{1, i}+T_{4, j}}
$$

where the $i, j$ represent the indexes of $\min _{1 \leq k \leq N}\left(T_{2, k} / \theta_{B}-\right.$ $\left.T_{1, k}\right)$ and $\min _{1 \leq k \leq N}\left(T_{4, k}-T_{3, k} / \theta_{B}\right)$, respectively, intersecting at the maximum $\hat{d}$ (which is the uppermost vertex shown as point A in Fig. 4). Note that in order to find this MLE, a total number of $N^{2}$ intersections have to be compared.

When $T_{2, k} \neq T_{3, k}$, for some $k$, the problem becomes a little involved. From Lemma 1, we know that $\hat{d}$ lies somewhere on the boundary of the support region. Notice further that according to (10) in order to minimize $\xi$ it is necessary to select $d$ as large as possible and $\theta_{B}$ as small as possible. 
Suppose that $\hat{d}$ lies on $\min _{1 \leq k \leq N}\left(T_{2, k} / \theta_{B}-T_{1, k}\right)$ and let $i=$ $\arg \min _{1 \leq k \leq N}\left(T_{2, k} / \theta_{B}-T_{1, k}\right)$ corresponding to the maximum $d$ (i.e., point $\mathrm{A}$ in Fig. 4), then from (10) $\xi$ can be written as

$$
\begin{aligned}
\xi & =\sum_{k=1}^{N} \frac{T_{2, k}-T_{3, k}}{\theta_{B}}-\sum_{k=1}^{N}\left(T_{1, k}-T_{4, k}\right)-2 N\left(\frac{T_{2, i}}{\theta_{B}}-T_{1, i}\right) \\
& =\frac{1}{\theta_{B}} \sum_{k=1}^{N}\left(T_{2, k}-T_{3, k}-2 T_{2, i}\right)-\sum_{k=1}^{N}\left(T_{1, k}-T_{4, k}-2 T_{1, i}\right) .
\end{aligned}
$$

Since the term $\sum_{k=1}^{N}\left(T_{2, k}-T_{3, k}-2 T_{2, i}\right)$ is always negative, $\xi$ can be minimized by taking $\theta_{B}$ as small as possible on $\min _{1 \leq k \leq N}\left(T_{2, k} / \theta_{B}-T_{1, k}\right)$. Hence, $\hat{d}$ and $\hat{\theta}_{B}$ in this general case are equal to or less than the MLE given by (23) and (24), respectively (i.e., either on point A shown in Fig. 4 or to the left of it). An alternative justification for the fact that $\hat{d}$ and $\hat{\theta}_{B}$ are equal to or less than the MLE given by (23) and (24), respectively, is to assume by contradiction that $\hat{d}$ lies on $\min _{1 \leq k \leq N}\left(T_{2, k} / \theta_{B}-T_{1, k}\right)$, with $i=\arg \min _{1 \leq k \leq N}\left(T_{2, k} / \theta_{B}-T_{1, k}\right)$, which does not correspond to the maximum $d$ (i.e., not on the curve passing through point $\mathrm{A}$ in Fig. 4). According to (25), $\xi$ is minimized by choosing $\theta_{B}$ as small as possible. Taking into account the continuity of $\xi$ with respect to $\theta_{B}$ and $d$, one can show that $\xi$ is monotonically decreasing as long as $\theta_{b}$ is decreased until it reaches the value corresponding to the point $\mathrm{A}$.

Now suppose that $\hat{d}$ lies on $\min _{1 \leq k \leq N}\left(T_{4, k}-T_{3, k} / \theta_{B}\right)$ and let $j=\arg \min _{1 \leq k \leq N}\left(T_{4, k}-T_{3, k} / \theta_{B}\right)$ corresponding to the maximum $d$ (i.e., point $\mathrm{A}$ in Fig. 4), then $\xi$ can be written as

$$
\begin{aligned}
\xi & =\sum_{k=1}^{N} \frac{T_{2, k}-T_{3, k}}{\theta_{B}}-\sum_{k=1}^{N}\left(T_{1, k}-T_{4, k}\right)-2 N\left(T_{4, j}-\frac{T_{3, j}}{\theta_{B}}\right) \\
& =\frac{1}{\theta_{B}} \sum_{k=1}^{N}\left(T_{2, k}-T_{3, k}+2 T_{3, j}\right)-\sum_{k=1}^{N}\left(T_{1, k}-T_{4, k}+2 T_{4, j}\right) .
\end{aligned}
$$

From (26), it is clear that $\xi$ can be minimized by taking the largest possible $\theta_{B}$ if $\sum_{k=1}^{N}\left(T_{2, k}-T_{3, k}+2 T_{3, j}\right)$ is positive and by taking the smallest possible $\theta_{B}$ if $\sum_{k=1}^{N}\left(T_{2, k}-T_{3, k}+2 T_{3, j}\right)$ is negative as depicted by Fig. 4. Hence, for

$$
2 N T_{3, j}>\sum_{k=1}^{N}\left(T_{3, k}-T_{2, k}\right)
$$

MLE is again given by (23) and (24). And for

$$
2 N T_{3, j}<\sum_{k=1}^{N}\left(T_{3, k}-T_{2, k}\right)
$$

MLE is given by the intersection of the curves $\left(T_{4, m}-T_{3, m} \theta_{B}\right)$ and $\left(T_{4, n}-T_{3, n} / \theta_{B}\right)$ (denoting the intersections of the curves
$d=T_{4, k}-T_{3, k} / \theta_{B}$ and $d=T_{4, l}-T_{3, l} / \theta_{B}$ as $d^{k, l}$, and $d^{k, l}$ satisfy the constraints (8) and (9)), where

$$
\begin{array}{r}
(m, n)=\arg \max _{k, l}\left\{d^{k, l} \mid 2 N T_{3, k}<\sum_{r=1}^{N}\left(T_{3, r}-T_{2, r}\right) ;\right. \\
\left.2 N T_{3, l}>\sum_{r=1}^{N}\left(T_{3, r}-T_{2, r}\right)\right\} .
\end{array}
$$

Basically, the indexes $(m, n)$ in (27) identify the first vertex of the support region located to the left of the vertex A for which a change of sign occurs in $2 N T_{3, n}-\sum_{r=1}^{N}\left(T_{3, r}-T_{2, r}\right)$. In Fig. 4, this vertex is represented by the point $\mathrm{B}$, and the MLE $\left(\hat{d}, \hat{\theta}_{B}\right)$ in this case is given by

$$
\hat{d}=T_{4, m}-\frac{T_{3, m}\left(T_{4, m}-T_{4, n}\right)}{T_{3, m}-T_{3, n}}
$$

and

$$
\hat{\theta}_{B}=\frac{T_{3, m}-T_{3, n}}{T_{4, m}-T_{4, n}} .
$$

Lemma 3: To the left of the point where $\min _{1 \leq k \leq N}\left(T_{2, k} / \theta_{B}-T_{1, k}\right)$ and $\min _{1 \leq k \leq N}\left(T_{4, k}-T_{3, k} / \theta_{B}\right)$ intersect (i.e., point $\mathrm{A}$ shown in Fig. 4), the boundary of the support region is formed by the curves $\left(T_{4, k}-T_{3, k} / \theta_{B}\right), k=1, \ldots, N$ in such a way that as $\theta_{B}$ increases, a curve $\left(T_{4, m}-T_{3, m} / \theta_{B}\right)$ forms the new boundary of the support region after intersecting the curve $\left(T_{4, n}-T_{3, n} / \theta_{B}\right)$ if and only if $m<n$.

Proof: The curve $\left(T_{4, N}-T_{3, N} / \theta_{B}\right)$ starts as the most negative for small $\theta_{B}$ and ends up as the largest positive asymptotically approaching $T_{4, N}$ as $\theta_{B}$ increases. Similarly, the curve $\left(T_{4,1}-T_{3,1} / \theta_{B}\right)$ starts as the least negative for small $\theta_{B}$ and ends up as the smallest positive asymptotically approaching $T_{4,1}$ as $\theta_{B}$ increases. All the curves $\left(T_{4, k}-T_{3, k} / \theta_{B}\right) k=1, \ldots, N$, are arranged in descending order for small $\theta_{B}$ and in ascending order for large $\theta_{B}$ and they intersect each other somewhere around the true value of $\theta_{B}$. Since the slope of each curve $\left(T_{4, k}-T_{3, k} / \theta_{B}\right), k=1, \ldots, N$ is $T_{3, k} / \theta_{B}^{2}$, the slope of the curve with index $m$ is lesser than the slope of the curve with index $n$ if $m<n$. Therefore, as $\theta_{B}$ increases, a curve can form the new boundary of the support region by intersecting another curve only if its index is lower than the previous one.

Lemma 4: The MLE $\left(\hat{d}, \hat{\theta}_{B}\right)$, whether (23) and (24) or (28) and (29), is unique.

Proof: Note that the likelihood function is continuous on the boundary of the support region because different curves intersect each other on the vertices due to which there will be no jumps in $\xi$ and subsequently in the likelihood function. Now considering the fact that $2 N T_{3, j}>\sum_{k=1}^{N}\left(T_{3, k}-T_{2, k}\right)$ for $j=N$, let

$$
q=\arg \max _{j}\left\{T_{3, j} \mid 2 N T_{3, j}<\sum_{k=1}^{N}\left(T_{3, k}-T_{2, k}\right)\right\} .
$$


Then it must also be true that $2 N T_{3, j}<\sum_{k=1}^{N}\left(T_{3, k}-T_{2, k}\right)$ $\forall j<q$, i.e., for $j=1, \ldots, q-1$ and $2 N T_{3, j}>\sum_{k=1}^{N}\left(T_{3, k}-\right.$ $\left.T_{2, k}\right) \forall j>q$, i.e., for $j=q+1, \ldots, N$. Fig. 3 shows the sign of the term $2 N T_{3, j}-\sum_{k=1}^{N}\left(T_{3, k}-T_{2, k}\right)$ for each $j=1, \ldots, N$. There will always be just one change, if any, in the sign of this term from positive to negative. Therefore, $\xi$ can be minimized by making $\theta_{B}$ as large as possible on $\left(T_{4, q+1}-T_{3, q+1} / \theta_{B}\right)$ and as small as possible on the curve $\left(T_{4, q}-T_{3, q} / \theta_{B}\right)$ (or on $\left(T_{2, i} / \theta_{B}-T_{1, i}\right)$ if there is no such $\left.q\right)$ as shown in Fig. 3.

This fact, combined with Lemma 3, proves that the intersection of the curves forming the MLE is always unique.

Lemma 5: Of all the intersections of $\left(T_{2, k}-\theta_{A}\right) /\left(T_{1, k}+d\right)$ with $\left(T_{3, k}-\theta_{A}\right) /\left(T_{4, k}-d\right)$, only two points satisfy the constraints (11) and (12) in a way that they represent the starting and ending points of the support region and the point with minimum $\theta_{B}$ is the one with maximum $\theta_{A}$.

Proof: Consider the curves $\left(T_{2, k}-\psi\right) /\left(T_{1, k}+d\right)$ with $\left(T_{3, k}-\psi\right) /\left(T_{4, k}-d\right)$ as a function of $\psi$ in order to avoid confusion between the actual unknown parameter $\theta_{A}$ and the variable with respect to which the above functions are drawn. Now utilizing (1) and (2), we can write

$$
\begin{aligned}
& \frac{T_{2, k}-\psi}{T_{1, k}+d}=\left(1+\frac{X_{k}}{T_{1, k}+d}\right) \theta_{B}+\frac{\theta_{A}-\psi}{T_{1, k}+d}, \\
& \frac{T_{3, k}-\psi}{T_{4, k}-d}=\left(1-\frac{Y_{k}}{T_{4, k}-d}\right) \theta_{B}+\frac{\theta_{A}-\psi}{T_{4, k}-d}, \ldots, N \\
& k=1, \ldots, N .
\end{aligned}
$$

It is clear that when $\psi=\theta_{A},\left(T_{2, k}-\psi\right) /\left(T_{1, k}+d\right)>$ $\left(T_{3, k}-\psi\right) /\left(T_{4, k}-d\right) \forall k$. Therefore, a support region does exist where the constraints (11) and (12) are satisfied. Now the slopes and y-intercepts of the straight lines $\left(T_{2, k}-\psi\right) /\left(T_{1, k}+d\right)$ are $-\left(T_{1, k}+d\right)^{-1}$ and $\left[1+X_{k}\left(T_{1, k}+d\right)^{-1}\right] \theta_{B}+\theta_{A}\left(T_{1, k}+d\right)^{-1}$ respectively, and the slopes and y-intercepts of the straight lines $\left(T_{3, k}-\psi\right) /\left(T_{4, k}-d\right)$ are $-\left(T_{4, k}-d\right)^{-1}$ and $\left[1-Y_{k}\left(T_{4, k}-\right.\right.$ $\left.d)^{-1}\right] \theta_{B}+\theta_{A}\left(T_{4, k}-d\right)^{-1}$ respectively. The y-intercepts can attain any value depending on the random delays $X_{k}, Y_{k}$ and the sign and magnitude of $\theta_{A}$, but there is a set pattern in the slopes of these lines. According to the model (see Fig. 1), it is always true that

$$
\begin{aligned}
\left(T_{1,1}+d\right)^{-1}>\left(T_{4,1}-d\right)^{-1}>\left(T_{1,2}+d\right)^{-1}>\ldots \ldots \\
>\left(T_{4, N-1}-d\right)^{-1}>\left(T_{1, N}+d\right)^{-1}>\left(T_{4, N}-d\right)^{-1} .
\end{aligned}
$$

This is because $T_{1,1}<T_{4,1}<T_{1,2}<\ldots \ldots<T_{4, N-1}<$ $T_{1, N}<T_{4, N}$. Due to the alternating slopes, the lines $\left(T_{2, k}-\right.$ $\psi) /\left(T_{1, k}+d\right)$ and $\left(T_{3, k}-\psi\right) /\left(T_{4, k}-d\right)$ for every $k$ intersect each other on at least one point. According to the order of the slopes, both to the left and right of $\psi=\theta_{A}$, the support region ends after the first intersection. Therefore, there are exactly two points, $\left(\theta_{A}^{\prime}, \theta_{B}^{\prime}\right)_{1}$ and $\left(\theta_{A}^{\prime}, \theta_{B}^{\prime}\right)_{2}$, which define the starting and ending point of the support region. In addition, the point corresponding to minimum $\theta_{B}^{\prime}$ is the one with maximum $\theta_{A}^{\prime}$ since all the straight lines always have negative slopes.

\section{REFERENCES}

[1] S. Ganeriwal, R. Kumar, and M. B. Srivastava, "Timing synch protocol for sensor networks," in Proc. 1st Int. Conf. Embedded Network Sensor Systems, 2003, pp. 138-149, ACM Press.

[2] D. R. Jeske, "On the maximum likelihood estimation of clock offset," IEEE Trans. Commun., vol. 53, no. 1, pp. 53-54, Jan. 2005.

[3] I. F. Akyildiz, W. Su, Y. Sankarasubramaniam, and E. Cayirci, "Wireless sensor networks: A survey," Comput. Netw., vol. 38, no. 4, pp. 393-422, Mar. 2002.

[4] B. M. Sadler, "Fundamentals of energy-constrained sensor network systems," IEEE Aerosp. Electron. Syst. Mag. (Tutorials II), vol. 20, no. 8, pp. 17-35, Aug. 2005.

[5] B. M. Sadler, "Critical issues in energy-constrained sensor networks: Synchronization, scheduling, and acquisition," in Proc. IEEE Int. Conf. Acoust., Speech, Signal Process. (ICASSP), Philadelphia, PA, 2005, vol. 5, pp. 785-788.

[6] B. M. Sadler and A. Swami, "Synchronization in sensor networks: An overview," presented at the IEEE Military Commun. Conf. (MILCOM), Washington, DC, 2006.

[7] B. Sundararaman, U. Buy, and A. D. Kshemkalyani, "Clock synchronization for wireless sensor networks: A survey," Ad-Hoc Netw., vol. 3, no. 3, pp. 281-323, Mar. 2005.

[8] H. S. Abdel-Ghaffar, "Analysis of synchronization algorithm with time-out control over networks with exponentially symmetric delays," IEEE Trans. Commun., vol. 50, pp. 1652-1661, Oct. 2002.

[9] V. Paxson, "On calibrating measurements of packet transit times," in Proc. 7th ACM Sigmetrics Conf., Jun. 1998, vol. 26, pp. 11-21.

[10] D. Mills, "Internet time synchronization: The network time protocol," IEEE Trans. Commun., vol. 39, no. 10, pp. 1482-1493, Oct. 1991.

[11] J. Elson, L. Girod, and D. Estrin, "Fine-grained network time synchronization using reference broadcasts," in Proc. 5th Symp. Operating System Design Implementation, Boston, MA, Dec. 2002, pp. 147-163.

[12] M. Maroti, B. Kusy, G. Simon, and A. Ledeczi, "The flooding time synchronization protocol," in Proc. 2nd Int. Conf. Embedded Networked Sensor Systems, 2004, pp. 39-49.

[13] A. Syed and J. Heidemann, "Time synchronization for high latency acoustic networks," Tech. Rep. ISI-TR-2005-602, Apr. 2005.

[14] K. Noh, Q. Chaudhari, E. Serpedin, and B. Suter, "Novel clock phase offset and skew estimation two-way timing message exchanges for wireless sensor networks," IEEE Trans. Commun., vol. 55, no. 4, pp. 766-777, Apr. 2007.

[15] A. Papoulis, Probability, Random Variables and Stochastic Processes, 3rd ed. New York: McGraw-Hill, 1991.

[16] A. Leon-Garcia, Probability and Random Processes for Electrical Engineering, 2nd ed. Reading, MA: Addison-Wesley, 1993.

[17] S. Narasimhan and S. S. Kunniyur, "Effect of network parameters on delay in wireless ad hoc networks," University of Pennsylvania, Philadelphia, Tech. Rep., Jun. 2004.

[18] C. Bovy, H. Mertodimedjo, G. Hooghiemstra, H. Uijterwaal, and P. Mieghem, "Analysis of end-to-end delay measurements in internet," presented at the Passive and Active Measurements Workshop, Fort Collins, CO, Mar. 2002, presented at.

[19] S. Moon, P. Skelley, and D. Towsley, "Estimation and removal of clock skew from network delay measurements," presented at the IEEE INFOCOM Conf. Computer Communications, New York, Mar. 1999.

[20] S. M. Kay, Fundamentals of Statistical Signal Processing, Vol. I. Estimation Theory. Englewood Cliffs, NJ: Prentice-Hall, 1993.

[21] G. Pottie and W. Kaiser, "Wireless integrated network sensors," Commun. ACM, vol. 43, no. 5, pp. 51-58, May 2000.

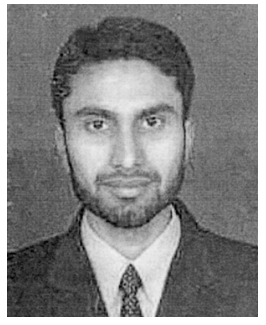

Qasim M. Chaudhari received the B.E. degree in electrical engineering from the National University of Sciences and Technology, Rawalpindi, Pakistan, in November 2001 and the M.S. degree in electrical engineering from the University of Southern California, Los Angeles, in May 2004. Since then, he is pursuing the Ph.D. degree in electrical engineering at Texas A\&M University, College Station.

He worked with in the SoC Tools group of Communications Enabling Technologies, Islamabad, Pakistan, from 2001 to 2002. Recently, he completed an internship with the HSDPA performance test team of Qualcomm Inc., San Diego, CA. His research interests include wireless communications in general and synchronization in wireless sensor networks in particular. 


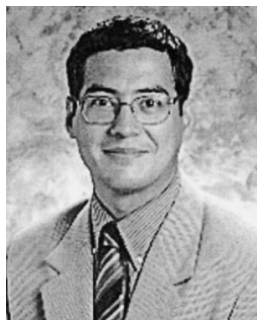

Erchin Serpedin received the Diploma degree (with highest distinction) in electrical engineering from the Polytechnic Institute of Bucharest, Bucharest, Romania, in 1991, the specialization degree in signal processing and transmission of information from Ecole Superieure D'Electricité, Paris, France, in 1992, the M.Sc. degree from the Georgia Institute of Technology, Atlanta, in 1992, and the Ph.D. degree in electrical engineering from the University of Virginia, Charlottesville, in January 1999.

In July 1999, he joined Texas A\&M University in College Station, as an Assistant Professor, where he currently holds the position of Associate Professor. His research interests lie in the areas of signal processing, bioinformatics, and telecommunications.

Dr. Serpedin received the NSF Career Award in 2001, the CCCT 2004 Best Conference Award, the Outstanding Faculty Award in 2004, the NRG Fellow Award in 2005, the TEES Award in 2005, and the ASEE Fellow Award in 2006. $\mathrm{He}$ is currently serving as an Associate Editor for the IEEE COMMUNICATIONS LetTers, the IEEE TRAnsactions on Signal Processing, the IEEE TRANSACTIONS ON COMMUNICATIONS, the IEEE TRANSACTIONS ON WIRELESS COMMUNICATIONS, the EURASIP Journal on Advances in Signal Processing, and the EURASIP Journal on Bioinformatics and Systems Biology. He served also as a Technical Co-Chair of the Communications Theory Symposium at Globecom 2006 Conference, VTC Fall 2006: Wireless Access Track, and as Editor for the IEEE SIGNAL PROCESSING LETTERS.

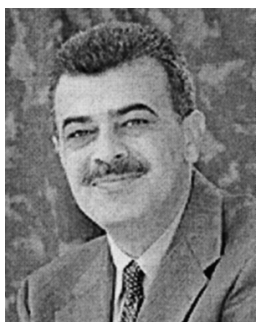

Khalid Qaraqe (M'97-S'00) was born in Bethlehem. He received the B.S. degree (with honors) in electrical engineering from the University of Technology, Baghdad, Iraq, in 1986, the M.S. degree in electrical engineering from the University of Jordan, Jordan, in 1989, and the Ph.D. degree in electrical engineering from Texas A\&M University, College Station, in 1997.

From 1989 to 2004, he held a variety of positions in many companies. He has more than 12 years of experience in the telecommunication industry. He has worked for Qualcomm, Enad Design Systems, Cadence Design Systems/Tality Corporation, STC, SBC, and Ericsson. He has worked on numerous GSM, CDMA, and WCDMA projects and has experience in product development, design, deployments, testing and integration. He joined the Department of Electrical Engineering of Texas A\&M University, Qatar, in July 2004, where he is now a Visiting Associate Professor. His research interests include communication theory and its application to design and performance, analysis of cellular systems, and indoor communication systems. Particular interests are in the development of 3G WCDMA wireless communications and diversity techniques. 